Research Article

\title{
Nonlinear Dynamic Analysis of Rotor Rub-Impact System
}

\author{
Youfeng Zhu $\mathbb{D},{ }^{1}$ Zibo Wang $\mathbb{D},{ }^{1}$ Qiang Wang, ${ }^{1}$ Xinhua Liu $\mathbb{D},{ }^{1}$ Hongyu Zang $\mathbb{D}{ }^{2}$ \\ and Liang Wang $\mathbb{D}^{3}$ \\ ${ }^{1}$ College of Transportation, Shandong University of Science and Technology, Qingdao 266590, China \\ ${ }^{2}$ College of Mechatronic Engineering, Shandong University of Science and Technology, Qingdao 266590, China \\ ${ }^{3}$ Shandong Sinogold New Energy Automotive Engineering Research Institute, Zibo 255000, China
}

Correspondence should be addressed to Youfeng Zhu; zhuyf1976@163.com

Received 10 April 2019; Accepted 16 July 2019; Published 29 November 2019

Academic Editor: Francesco Pellicano

Copyright (C) 2019 Youfeng Zhu et al. This is an open access article distributed under the Creative Commons Attribution License, which permits unrestricted use, distribution, and reproduction in any medium, provided the original work is properly cited.

A dynamic model of a double-disk rub-impact rotor-bearing system with rubbing fault is established. The dynamic differential equation of the system is solved by combining the numerical integration method with MATLAB. And the influence of rotor speed, disc eccentricity, and stator stiffness on the response of the rotor-bearing system is analyzed. In the rotor system, the time history diagram, the axis locus diagram, the phase diagram, and the Poincaré section diagram in different rotational speeds are drawn. The characteristics of the periodic motion, quasiperiodic motion, and chaotic motion of the system in a given speed range are described in detail. The ways of the system entering and leaving chaos are revealed. The transformation and evolution process of the periodic motion, quasiperiodic motion, and chaotic motion are also analyzed. It shows that the rotor system enters chaos by the way of the period-doubling bifurcation. With the increase of the eccentricity, the quasi-periodicity evolution is chaotic. The quasiperiodic motion evolves into the periodic three motion phenomenon. And the increase of the stator stiffness will reduce the chaotic motion period.

\section{Introduction}

In the previous study of rotor dynamics, the single-disc rotor system is the main research object. The single disk rotor system is a real simplification of the actual rotor system. Its structure is simple, and it can well reflect the dynamic characteristics of the actual system. Scholars at home and abroad have used this model to discover nonlinear phenomena in many rotor systems. Luo et al. [1] studied the complex motion of an elastic rotor system with coupling failures of a crack and a rubbing fault and pointed out that rubbing faults occupy a major position when the coupling faults of a crack and a rub impact occur at the same time. Muszynska [2] proposed a simplified nonlinear oil film model and used this model to analyze the stability of the rotor-bearing system. The model is built with an amount that characterizes the circumferential flow rate of the fluid. Capone $[3,4]$ proposed a nonlinear oil film force model under the assumption of correcting short bearings. This model is one of the commonly used oil film force models in dynamic analysis of rotor systems and has good accuracy and convergence. Jiang et al. [5] simplified the rotor system of the centrifugal pump impeller and established the vibration model of the unbalanced impeller rotor with loose support failure under the condition of the introduction of nonlinear lateral fluid excitation force. The effects of the lateral fluid excitation force and the bearing mass on the nonlinear dynamic characteristics of the system were analyzed. Zhang and Wen [6] had established a mathematical model with nonlinear oil film force for the loosening of the two ends of the bearing system in a rotor-bearing system. It is found that if the vibration displacement is less than the maximum gap, the frequency spectrum will have abundant low-frequency components except the frequency of one time. If the gap is larger than the maximum gap, the high-frequency composition of the highfrequency ratio will appear and the frequency of one time will disappear. Ma et al. [7] used the finite element method to establish a rotor system model with bearing failure. It was found that the basic stiffness has a significant influence on the nonlinear characteristics of the system.

However, in actual industrial production, there is often more than one rotor, and there may be a plurality of turntables or a plurality of rotor systems connected to each other by a coupling to form a multispan large rotor system. There are many studies on multiple turntables or multiple 

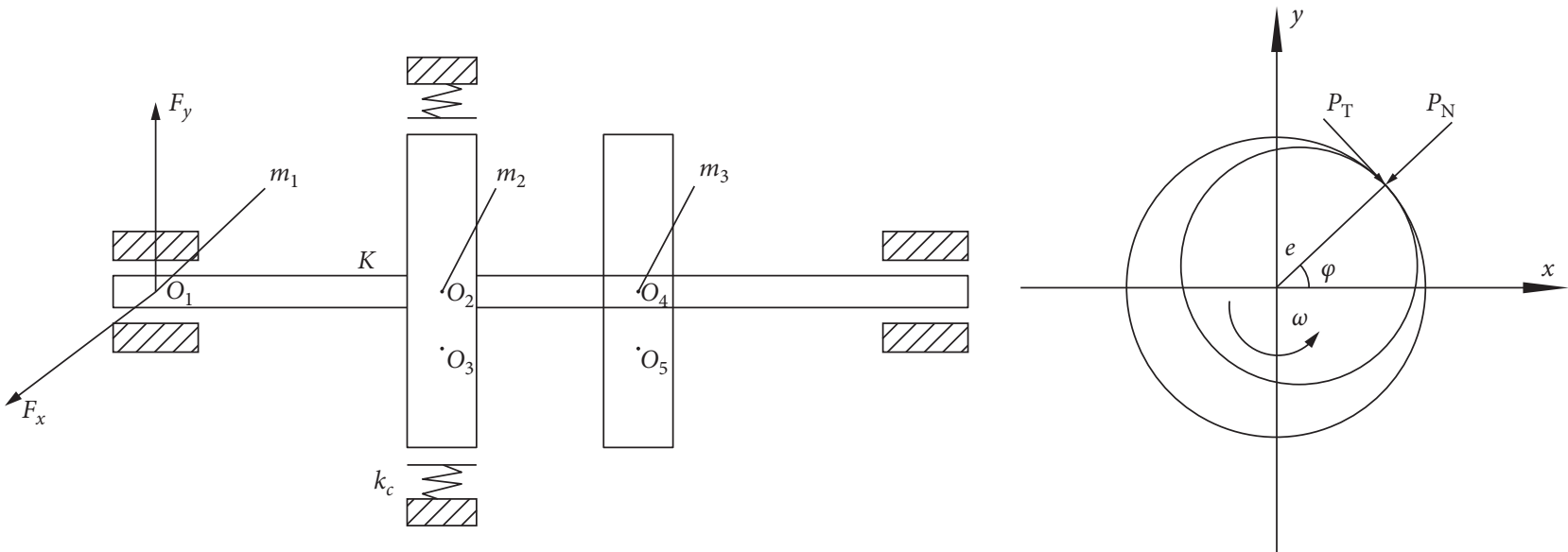

FIgure 1: (a) Mechanical model of the rotor system. (b) Model of rub impact.

rotors at home and abroad. Luo et al. [8] established a double-span rub-impact rotor-bearing system and conducted research on the two cases of single-disc and doubledisc rubbing. He and Yuan [9] reduced the aerogenerator rotor to a 22-DOF double-disk cantilever continuum rotor system and studied the influence of the speed and eccentricity on the system's rub-impact response. Ren et al. [10] used the finite element method to establish a double-disk rotor model with rub-lip crack coupling failure and pointed out that large rub-impact stiffness has a great influence on the system's instability speed and stability area. Ma et al. [11] used a finite element method to establish a dual-disk rotor model with fixed-point rubbing. The simulation results show that the gap between the disk and the rod and the contact stiffness has an important influence on the vibration strength and the impact spring back form. Hu and Hou [12] had established a dual disc-biased rotor system with rubimpact fault. The simulation results show that the eccentricity and stator stiffness can affect the stability of the system without affecting the oil film whirl. There are multiple frequency components in the system response. Different faults will interact and have a certain degree of self-healing ability. Luo et al. [13] studied the double-disc rotor with coupling failures of cracks and rubbing. The results show that there are many ways to enter chaos such as double period bifurcation, double period backward bifurcation, and Hopf bifurcation at different rotational speeds. Li et al. [14] considered the impact of the elastic support on the doublespan rotor system and compared it with the rigid support. It was found that the nonlinearity of the elastically supported rotor system was significantly enhanced and the chaotic region was increased. Liu et al. [15-17] established the rotorstator-bearing system mechanics model of rubbing faults and pointed out that rubbing and eccentricity are the main causes of system chaos. Rubbing will cause the system to generate reverse whirl, and the system enters chaos through quasi-periodicity bifurcation.

In this paper, a single-span double-disc rotor-bearing system model is established by considering the nonlinear bearing oil film force. The numerical analysis method is used to solve the differential equations of the system, and the nonlinear dynamic characteristics of the rotor system are analyzed. This provides a theoretical basis for the rotor system.

\section{Dynamic Model of Dual-Disk Rotor System}

As shown in Figure 1, the research object in this paper is a single-span double-disk model. Both ends of the shaft are supported by a pair of identical sliding bearings, assuming that there is no mass-elastic shaft between the rotor and the two bearings. $\mathrm{O}_{1}$ is the geometric center of the bearing bush. $\mathrm{O}_{2}$ and $\mathrm{O}_{3}$ are the geometric centers of the two rotor discs. $\mathrm{O}_{4}$ and $\mathrm{O}_{5}$ are the centroids of the two rotor discs. The concentrated mass of the shaft at the bearing is $m_{1}$, and the damping is $c_{1}$. The concentrated mass of the left-end rotor disc at the shaft is $m_{2}$, the damping is $c_{2}$, and the eccentricity is $b_{1}$. The concentrated mass of the right rotor disc at the axis of rotation is $m_{3}$, the damping is $c_{3}$, and the eccentricity is $b_{2} . k$ and $k_{c}$ are the stiffness of the rotor shaft and stator. $F_{x}$ and $F_{y}$ are the components of nonlinear film forces in the $x$ and $y$ directions. The plain bearing has a bearing radius of $R$, a bearing length of $L$, and an oil film gap of $c$.

\section{The Differential Equation of Motion for Rotor System}

The local friction model of the rotating stator is shown in Figure $1(\mathrm{~b})$, where $P_{\mathrm{N}}$ and $P_{\mathrm{T}}$ are the component forces of the rubbing force in the radial direction and the normal direction, respectively. $\varphi$ is the angle between the normal of the rubbing point and the $x$-axis. For the convenience of research, the following assumptions are made in the process of establishing the model: the friction between the rotor and the stator satisfies the Coulomb friction law, and it is considered that the two are elastic collisions and the deformation is elastic deformation. Then, $P_{\mathrm{N}}$ and $P_{\mathrm{T}}$ can be expressed as

$$
\left\{\begin{array}{l}
P_{\mathrm{N}}=k_{c}(e-\delta), \\
P_{\mathrm{T}}=f \cdot P_{\mathrm{N}}, \\
(e \geq \delta),
\end{array}\right.
$$


where $f$ is the coefficient of friction and $e$ is the radial displacement of the geometric center of the disk:

$$
e=\sqrt{x^{2}+y^{2}} .
$$

Decompose the rubbing force into the $x$ and $y$ directions:

$$
\left\{\begin{array}{c}
P_{x} \\
P_{y}
\end{array}\right\}=\left(\begin{array}{cc}
-\cos \varphi & \sin \varphi \\
-\sin \varphi & \cos \varphi
\end{array}\right)\left\{\begin{array}{l}
P_{\mathrm{N}} \\
P_{\mathrm{T}}
\end{array}\right\} .
$$

Comprehending (1)-(3), rubbing can be expressed as [18]

$$
\left\{\begin{array}{c}
P_{x} \\
P_{y}
\end{array}\right\}=-\frac{(e-\delta) k_{c}}{e}\left[\begin{array}{cc}
1 & -f \\
f & 1
\end{array}\right]\left\{\begin{array}{l}
x \\
y
\end{array}\right\},
$$

where $x$ and $y$ represent the displacement components of the geometric center of the rotor disc, $\delta$ is the rotor gap, and $e$ is the radial displacement of the rotor. The radial and axial displacements of the left end of the rotor are, respectively, $x_{1}$ and $y_{1}$. The displacement of the left disc is $x_{2}$ and $y_{2}$. The displacement of the right disc is $x_{3}$ and $y_{3}$. The displacement of the right end of the rotor is $x_{4}$ and $y_{4}$. The rotor speed is $\omega$. The differential equations of motion of the rotor-bearing system with double disks rubbing against are given below:

$$
\begin{aligned}
m_{1} \ddot{x}_{1}+c_{1} \dot{x}_{1}+k\left(x_{1}-x_{2}\right) & =F_{x}, \\
m_{1} \ddot{y}_{1}+c_{1} \dot{y}_{1}+k\left(y_{1}-y_{2}\right) & =F_{y}-m_{1} g, \\
m_{2} \ddot{x}_{2}+c_{2} \dot{x}_{2}+k\left(2 x_{2}-x_{1}-x_{3}\right) & =m_{2} b_{1} \omega^{2} \cos (\omega t)+P_{x}, \\
m_{2} \ddot{y}_{2}+c_{2} \dot{y}_{2}+k\left(2 y_{2}-y_{1}-y_{3}\right) & =m_{2} b_{1} \omega^{2} \sin (\omega t)+P_{y}-m_{2} g, \\
m_{3} \ddot{x}_{3}+c_{3} \dot{x}_{3}+k\left(2 x_{3}-x_{2}-x_{4}\right) & =m_{3} b_{2} \omega^{2} \cos (\omega t), \\
m_{3} \ddot{y}_{3}+c_{3} \dot{y}_{3}+k\left(2 y_{3}-y_{2}-y_{4}\right) & =m_{3} b_{2} \omega^{2} \sin (\omega t)-m_{3} g, \\
m_{1} \ddot{x}_{4}+c_{1} \dot{x}_{4}+k\left(x_{4}-x_{3}\right) & =F_{x 1}, \\
m_{1} \ddot{y}_{4}+c_{1} \dot{y}_{4}+k\left(y_{4}-y_{3}\right) & =F_{y 1}-m_{1} g .
\end{aligned}
$$

Introducing dimensionless transformations

$$
\begin{aligned}
& \tau=\omega t, \\
& x_{1}=\frac{x_{1}}{c}, \\
& y_{1}=\frac{y_{1}}{c}, \\
& x_{2}=\frac{x_{2}}{c}, \\
& y_{2}=\frac{y_{2}}{c}, \\
& x_{3}=\frac{x_{3}}{c}, \\
& y_{3}=\frac{y_{3}}{c}, \\
& x_{4}=\frac{x_{4}}{c}, \\
& y_{4}=\frac{y_{4}}{c} .
\end{aligned}
$$

Substitute it into equation (3), and then the nondimensional differential equations for the motion of the twodisc rubbing rotor-bearing system are

$$
\begin{aligned}
\ddot{x}_{1}+\frac{c_{1}}{m_{1} \omega} \dot{x}_{1}+\frac{k}{m_{1} \omega^{2}}\left(x_{1}-x_{2}\right) & =\frac{s P}{m_{1} \omega^{2} c} f_{x}, \\
\ddot{y}_{1}+\frac{c_{1}}{m_{1} \omega} \dot{y}_{1}+\frac{k}{m_{1} \omega}\left(y_{1}-y_{2}\right) & =\frac{s P}{m_{1} \omega^{2} c} f_{y}-G, \\
\ddot{x}_{2}+\frac{c_{2}}{m_{2} \omega} \dot{x}_{2}+\frac{k}{m_{2} \omega^{2}}\left(2 x_{2}-x_{1}-x_{3}\right) & =\rho_{1} \cos (\tau)+\frac{P_{x}}{m_{2} \omega^{2} c}, \\
\ddot{y}_{2}+\frac{c_{2}}{m_{2} \omega} \dot{y}_{2}+\frac{k}{m_{2} \omega^{2}}\left(2 y_{2}-y_{1}-y_{3}\right) & =\rho_{1} \sin (\tau)+\frac{P_{y}}{m_{2} \omega^{2} c}-G, \\
\ddot{x}_{3}+\frac{c_{3}}{m_{3} \omega} \dot{x}_{3}+\frac{k}{m_{3} \omega^{2}}\left(2 x_{3}-x_{2}-x_{4}\right) & =\rho_{2} \sin (\tau), \\
\ddot{y}_{3}+\frac{c_{3}}{m_{3} \omega} y_{3}+\frac{k}{m_{3} \omega^{2}}\left(2 y_{3}-y_{2}-y_{4}\right) & =\rho_{2} \cos (\tau)-G, \\
\ddot{x}_{4}+\frac{c_{1}}{m_{1} \omega} \dot{x}_{4}+\frac{k}{m_{1} \omega^{2}}\left(x_{4}-x_{3}\right) & =\frac{s P}{m_{1} \omega^{2} c} f_{x 1}, \\
\ddot{y}_{4}+\frac{c_{1}}{m_{1} \omega} \dot{y}_{4}+\frac{k}{m_{1} \omega^{2}}\left(y_{4}-y_{3}\right) & =\frac{s P}{m_{1} \omega^{2} c} f_{y 1}-G .
\end{aligned}
$$

Introducing dimensionless transformations

$$
\begin{aligned}
\rho_{1} & =\frac{b_{1}}{c}, \\
\rho_{2} & =\frac{b_{2}}{c}, \\
G & =\frac{g}{c \omega^{2}}, \\
s & =\frac{\mu \omega R L}{P}\left(\frac{R}{c}\right)^{2}\left(\frac{L}{2 R}\right)^{2}, \\
P & =\frac{1}{2} m_{2}, \\
F & =s p \cdot f,
\end{aligned}
$$

where $\tau$ is the dimensionless time, $\rho_{1}$ and $\rho_{2}$ are the dimensionless eccentricity; $s$ is Sommerfeld's correction coefficients, and $f_{x}, f_{y}, f_{x 1}, f_{y 1}$ are dimensionless forms of nonlinear film forces.

\section{Nonlinear Dynamics Analysis of Rotor System}

The parameters of the dual-disk rotor system are shown in Table 1. In view of the strong nonlinearity of the film force, the numerical method is used to solve the differential equations in this paper. The integration step length is chosen as $T / 512$, and the error is set as $1 \times 10^{-6}$. In order to obtain a steady-state solution, the equation group is subjected to 
TABle 1: Parameters of the rotor system.

\begin{tabular}{lc}
\hline Parameter & Value \\
\hline$m_{1}$ & $4.0 \mathrm{~kg}$ \\
$m_{2}$ & $32.0 \mathrm{~kg}$ \\
$m_{3}$ & $32.0 \mathrm{~kg}$ \\
$K$ & $2.5 \times 10^{7} \mathrm{~N} \cdot \mathrm{m}^{-1}$ \\
$k_{c}$ & $1 \times 10^{7} \mathrm{~N} \cdot \mathrm{m}^{-1}$ \\
$c_{1}$ & $1050 \mathrm{~N} \cdot \mathrm{s} \cdot \mathrm{m}^{-1}$ \\
$c_{2}$ & $2100 \mathrm{~N} \cdot \mathrm{s} \cdot \mathrm{m}^{-1}$ \\
$b_{1}$ & $0.05 \mathrm{~mm}$ \\
$b_{2}$ & $0.05 \mathrm{~mm}$ \\
$\mu$ & $0.018 \mathrm{~Pa} \cdot \mathrm{s}$ \\
$R$ & $25 \mathrm{~mm}$ \\
$L$ & $12 \mathrm{~mm}$ \\
$c$ & $0.11 \mathrm{~mm}$ \\
$f$ & 0.1 \\
\hline
\end{tabular}

multiperiod numerical solution and the previous transient solution is discarded. In this section, bifurcation diagrams, time histories, axial trajectories, phase diagrams, and Poincaré cross sections are used to study the effects of rotor speed, rotor disc eccentricity, and stator stiffness variations on the nonlinear dynamic behavior of the system.

4.1. Effect of Rotation Speed on the Response of Rotor System. Figure 2 shows the response bifurcation diagram of the rotor system in the $x_{1}$ direction with the speed change. In the lowspeed region, 200-767 rad/s, the system performs quasiperiodic motion, in which the interval $200-513 \mathrm{rad} / \mathrm{s}$ is the cycle one motion and $514-766 \mathrm{rad} / \mathrm{s}$ is the cycle two motion. Subsequently, the system continuously generates bifurcations and finally enters chaotic motion at $\omega=767 \mathrm{rad} / \mathrm{s}$. With the increase of the speed, the rotor system deviates from the chaos interval to the cycle two movement when $\omega=984 \mathrm{rad} / \mathrm{s}$. After a brief period of four cycles, 1107-1146 $\mathrm{rad} / \mathrm{s}$, the rotor system becomes quasiperiodic motion and evolves into quasi-periodicity three motion at $\omega=1744 \mathrm{rad} /$ s. Finally, when $\omega=1855 \mathrm{rad} / \mathrm{s}$, the system enters a wide range of quasiperiodic motion intervals.

In order to show in detail the response characteristics of the system in the speed range $200-2500 \mathrm{rad} / \mathrm{s}$, in this section, multiple speeds are selected and the corresponding time histories, axes, phase diagrams, Poincaré sections, etc., are plotted to illustrate the system's motion.

When $\omega=300 \mathrm{rad} / \mathrm{s}$, the system makes a quasi-periodicity movement. At this time, the rotation speed is low, and the frictional force is small. The film force is the main factor affecting the system movement.

When the rotational speed increases to a certain value, the film force will generate a whirl to change the rotor system's motion state, and the system will produce double-cycled bifurcation from cycle one to cycle two. Figure 3 shows the response of the system at a rotational speed of $600 \mathrm{rad} / \mathrm{s}$. There are two peaks in the time history diagram. The axis trajectory is the two nested ellipses. The Poincare section is the two isolated points. At this point, the rotor system is in cycle two.

As the rotation speed continues to increase, the effect of oil film force begins to increase further. At this time, the oil

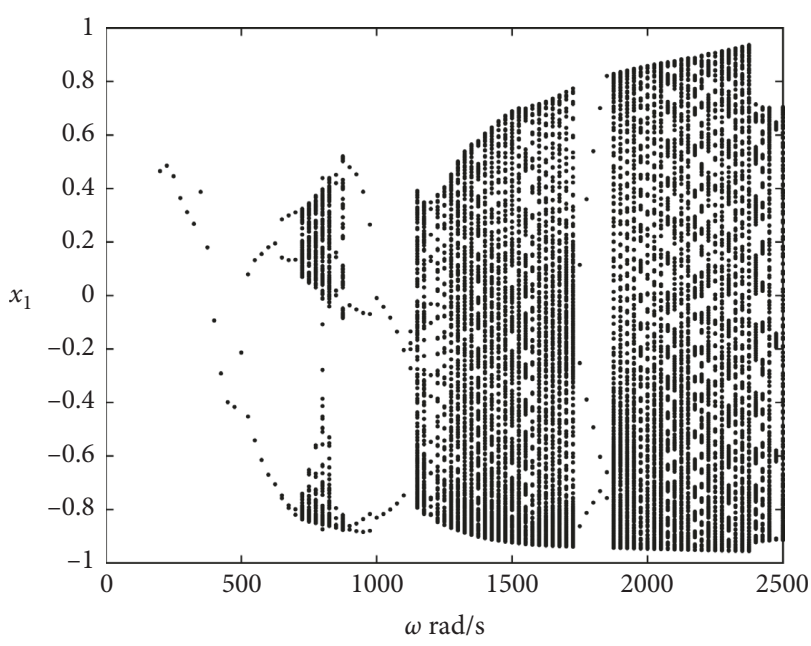

Figure 2: Bifurcation diagram of the rotor system in the $x_{1}$ direction.

film force is still the main factor affecting the stability of the rotor system. As the rotation speed increases, the whirlpool of the oil film increases and the rotor system becomes unstable. Figure 4 shows the system response when $\omega=750 \mathrm{rad} / \mathrm{s}$. There is no regularity in the time-domain graph. The Poincaré cross section is the two isolated islands. A series of noncoincident curves are located on the axis of the axis, and the system is chaotic. As the rotor speed increases further, the imbalance force due to eccentricity of the disc begins to gradually increase, and the system moves through the backward bifurcation regression cycle two.

Figure 5 shows the system response at $\omega=1400 \mathrm{rad} / \mathrm{s}$. With the increase of the rotational speed, the unbalanced force generated by the eccentricity of the disk in the rotor system also gradually increases. When it increases to a certain degree, the rotor motion state will change, and the system gradually evolves from the second cycle to the quasiperiodic motion. From the time history chart, it can be observed that the system has a "beat vibration" phenomenon. The Poincaré section is a closed curve. At this time, the system performs quasiperiodic motion.

When the speed increases to $1800 \mathrm{rad} / \mathrm{s}$, the system is a three-cycle motion. As the speed increases, the rotor system again enters the quasiperiodic motion after a brief period of three response intervals. Figure 6 shows the response of the rotor system at a speed of $2000 \mathrm{rad} / \mathrm{s}$. The Poincaré cross section is a closed curve composed of a series of points. At this time, the system rotor performs quasiperiodic motion. The axis trajectory is more compact and close to the limit circle at the bearing when compared to $\omega=1400 \mathrm{rad} / \mathrm{s}$.

\subsection{Effect of Eccentricity on Response of Rotor System.} Eccentricity often exists in the rotor system, and its main causes are structural design irrational, manufacturing or installation process errors, and material inhomogeneity. Eccentricity is the main source of unbalanced forces in the rotor system. From the differential equations, it can be seen that unbalanced forces have a great impact on the system. 


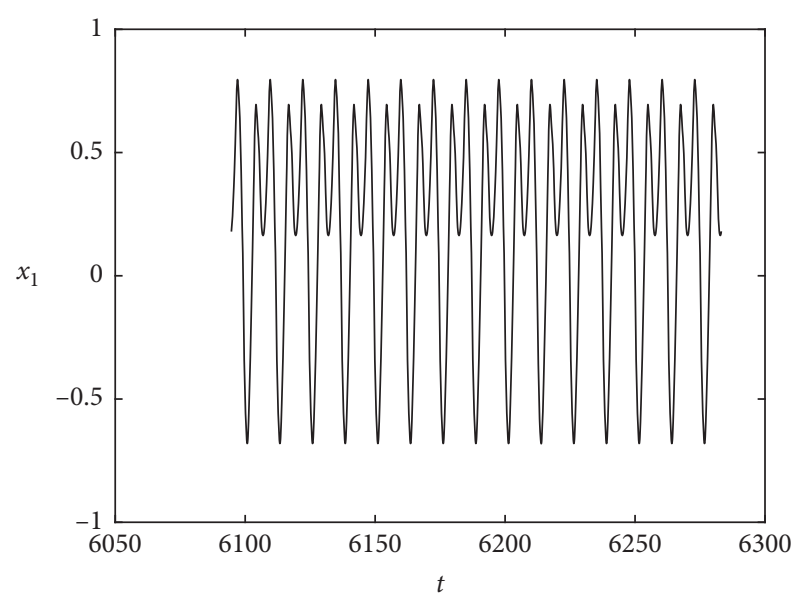

(a)

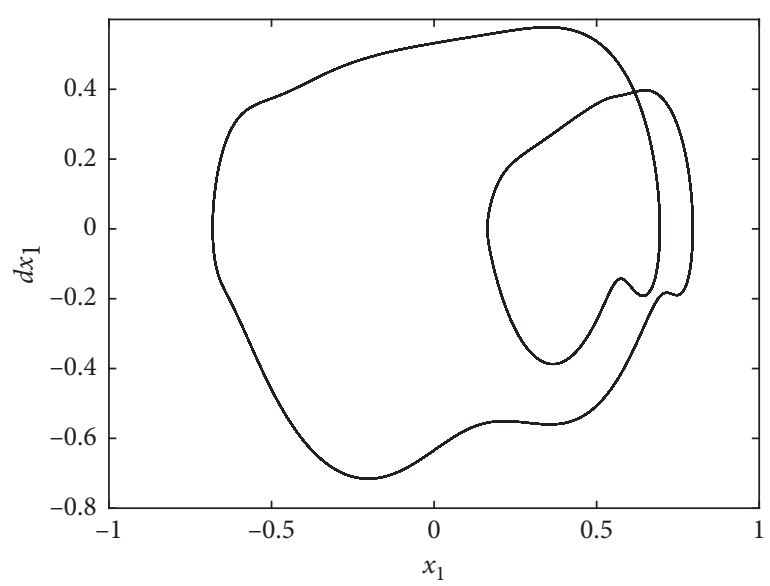

(c)

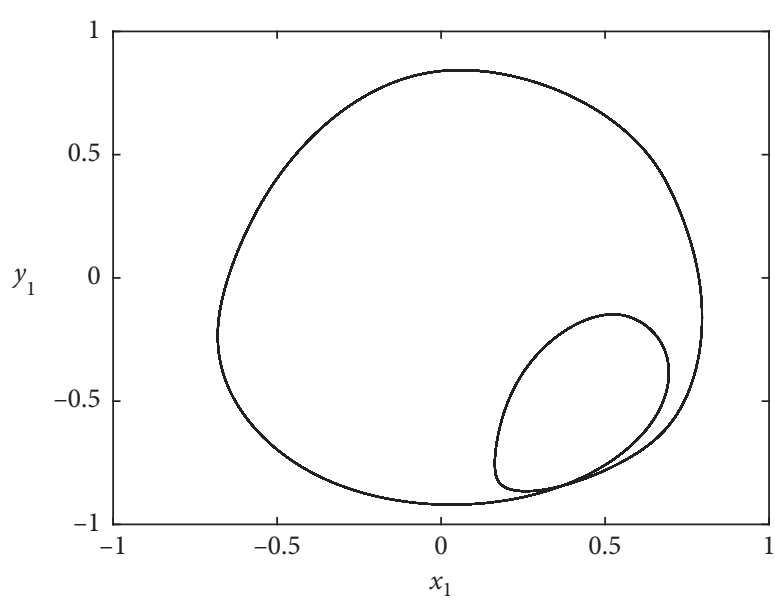

(b)

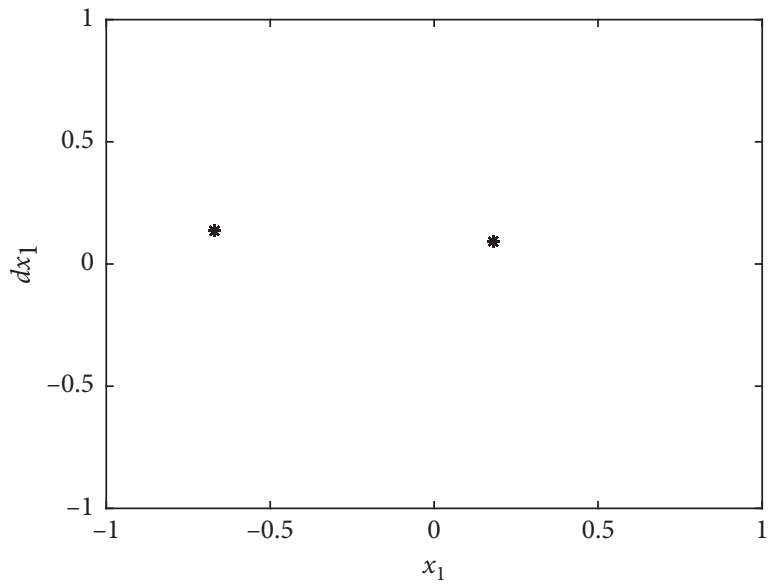

(d)

FIgURE 3: Response of the rotor system when $\omega=600 \mathrm{rad} / \mathrm{s}$.

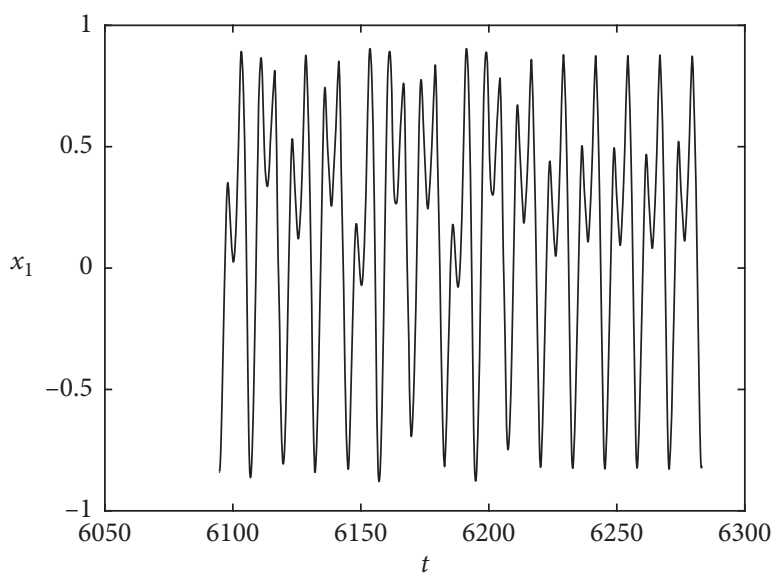

(a)

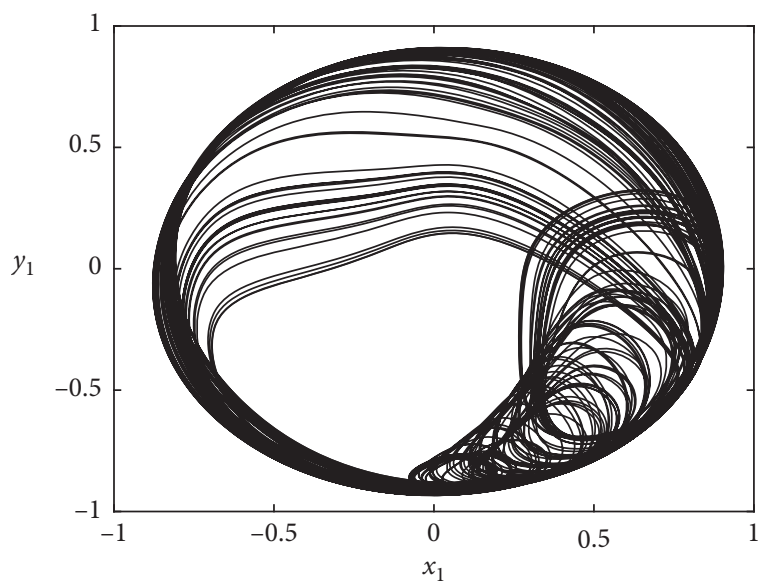

(b)

FIgURE 4: Continued. 


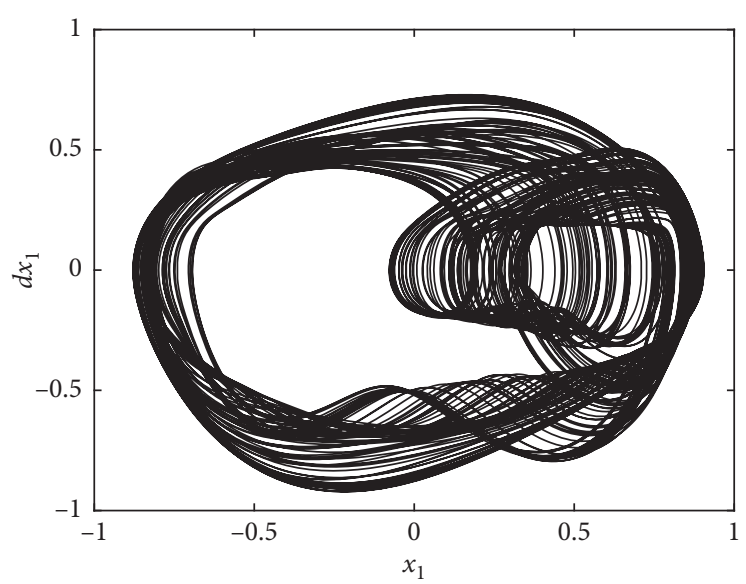

(c)

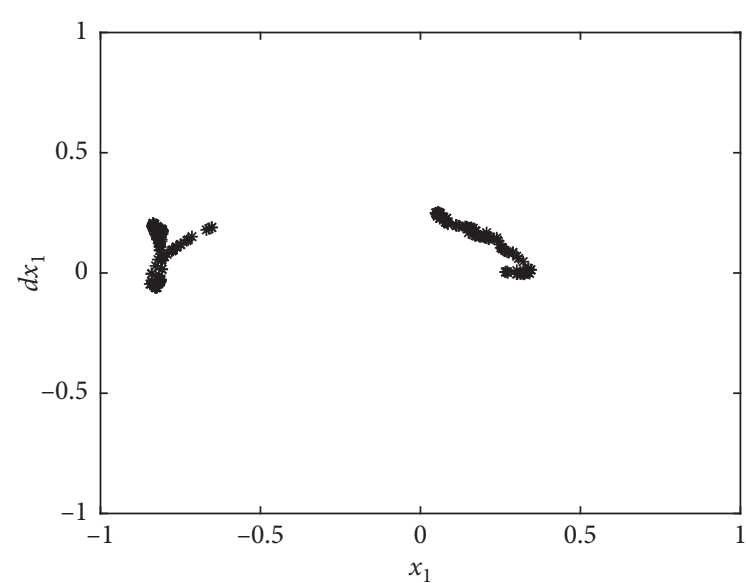

(d)

FIgURE 4: Response of the rotor system when $\omega=750 \mathrm{rad} / \mathrm{s}$.

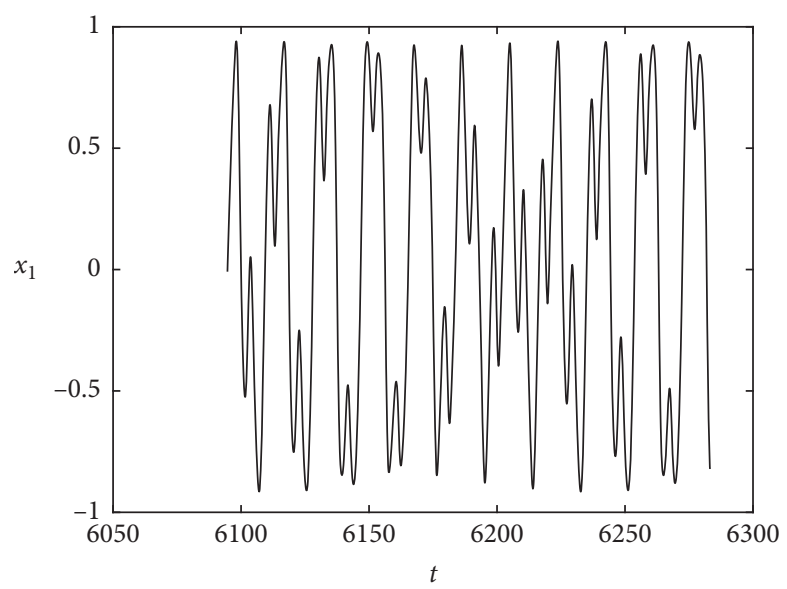

(a)

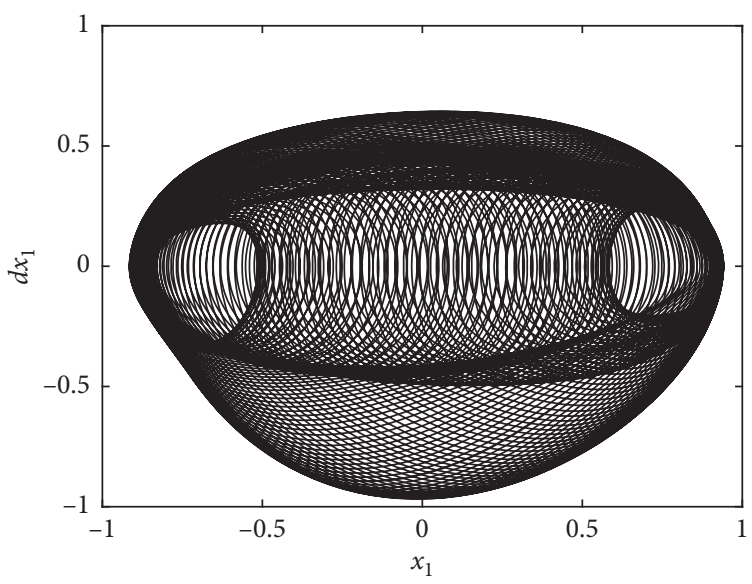

(c)

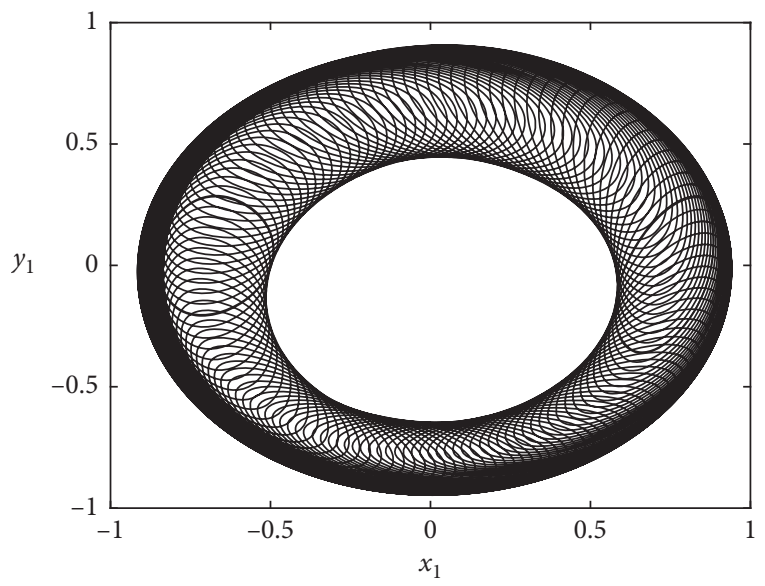

(b)

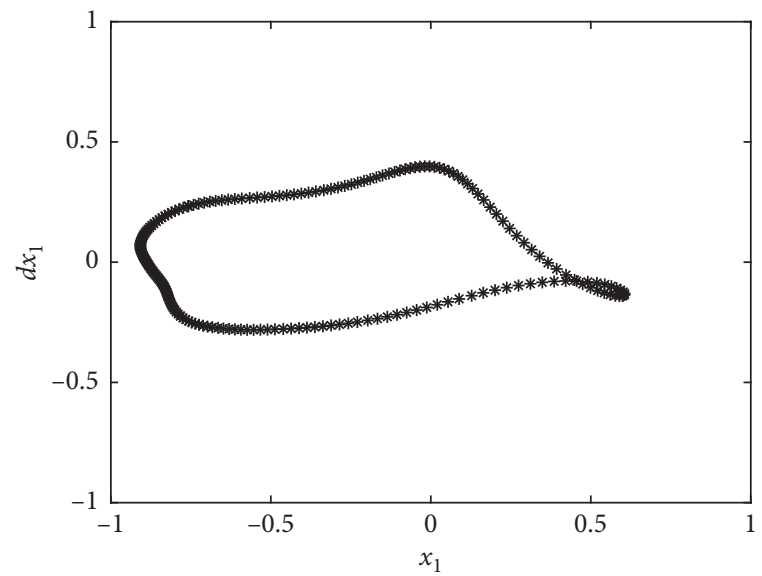

(d)

FIgURE 5: Response of the rotor system when $\omega=1400 \mathrm{rad} / \mathrm{s}$.

Under the same parameters, the eccentricity is different, and the magnitude of the imbalance force is different. The response of the system will also be very different. Therefore, it is of great significance to study the influence of eccentricity on rotor system response. In this section, the selected subrigidity is $1 \times 10^{7} \mathrm{~N} / \mathrm{m}$, and the eccentricity is the 


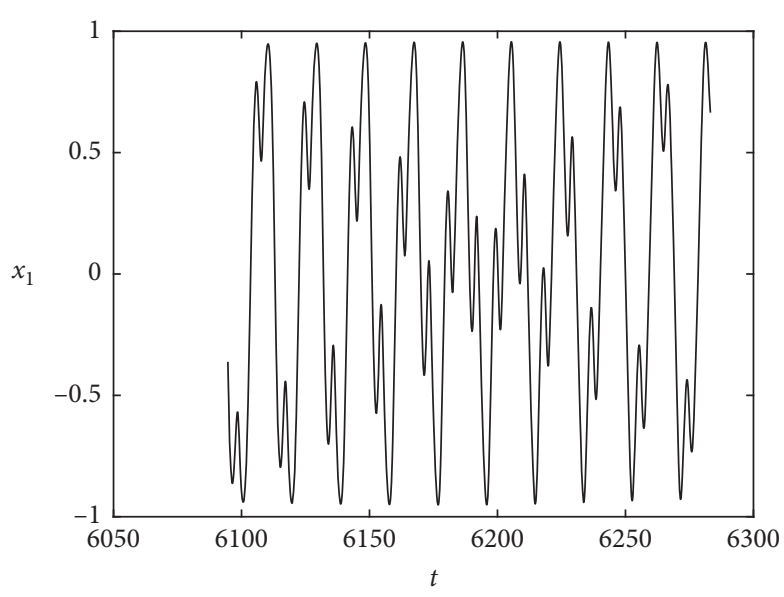

(a)

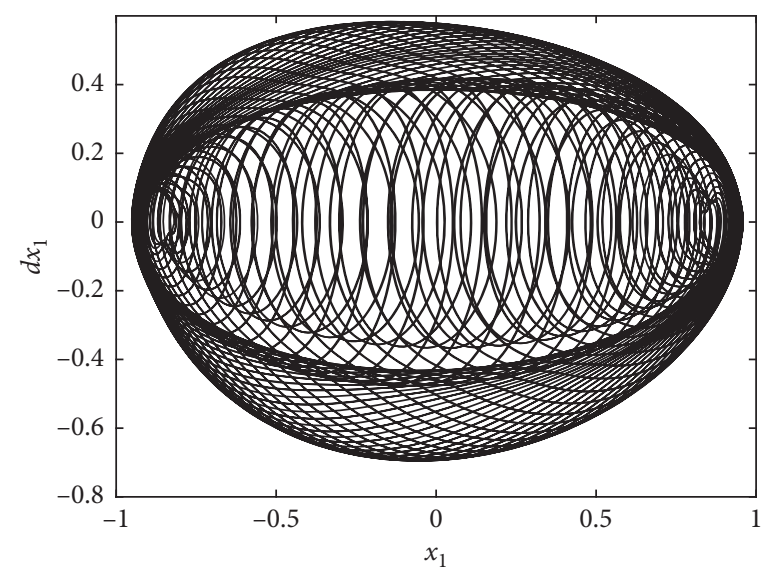

(c)

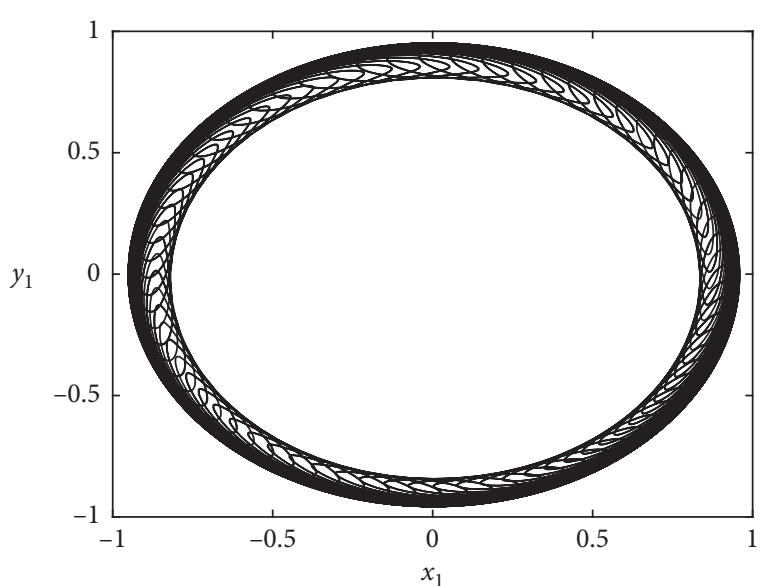

(b)

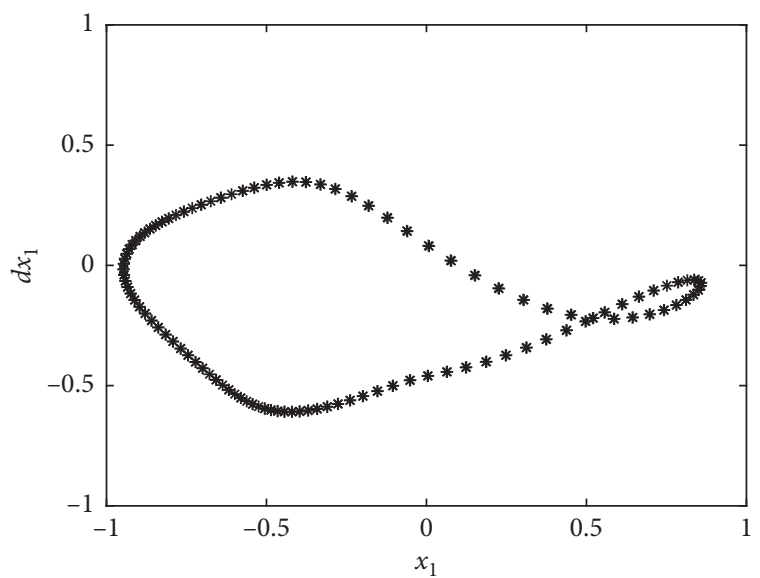

(d)

FIgURE 6: Response of the rotor system when $\omega=2000 \mathrm{rad} / \mathrm{s}$.

changing parameter, and the other parameters remain unchanged.

Figure 7 is the response bifurcation diagram of rotor system in the $x_{1}$ direction changing with the speed under the condition that $b_{1}=0.01 \mathrm{~mm}$ and other parameters remain unchanged. The movement of the system has experienced the cycle, almost the cycle, the P-K cycle, the quasi-periodicity cycle, the cycle, and the quasi-periodicity movement process.

When the rotation speed is low, the rotor system performs a stable quasiperiodic motion with an interval of $200-650 \mathrm{rad} / \mathrm{s}$, where the system is in cycle one at 200 $500 \mathrm{rad} / \mathrm{s}$. When the rotational speed reaches $500 \mathrm{rad} / \mathrm{s}$, the oil film vortex starts to occur, the system produces a perioddoubling bifurcation and a period two movement, and there are even peaks in the time history diagram. The Poincaré section is the two isolated points, as shown in Figure 8(a).

When the speed is greater than $650 \mathrm{rad} / \mathrm{s}$, the oil film whirl continues to increase, the system motion state continues to change and enters almost periodic motion state, as shown in Figure 8(b). There is a "clip" phenomenon in the time history diagram, and the Poincaré section is a closed curve formed by a point set. Figure 8(c) shows the response

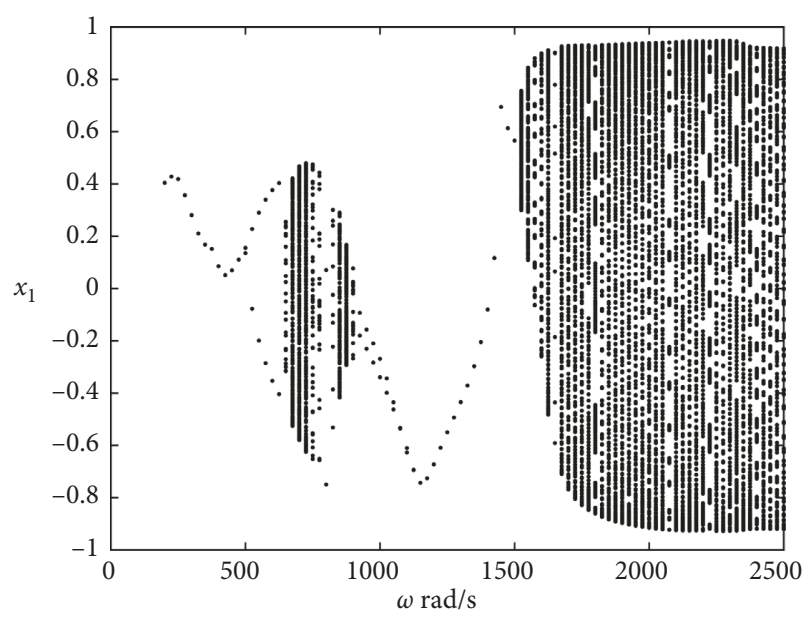

Figure 7: Bifurcation diagram of the rotor system in the $x_{1}$ direction when $b_{1}=0.01 \mathrm{~mm}$.

of the system at $\omega=825 \mathrm{rad} / \mathrm{s}$. The time-domain diagram shows that the system is a regular multicycle motion, and the Poincare section is 11 isolated points, and the system is 

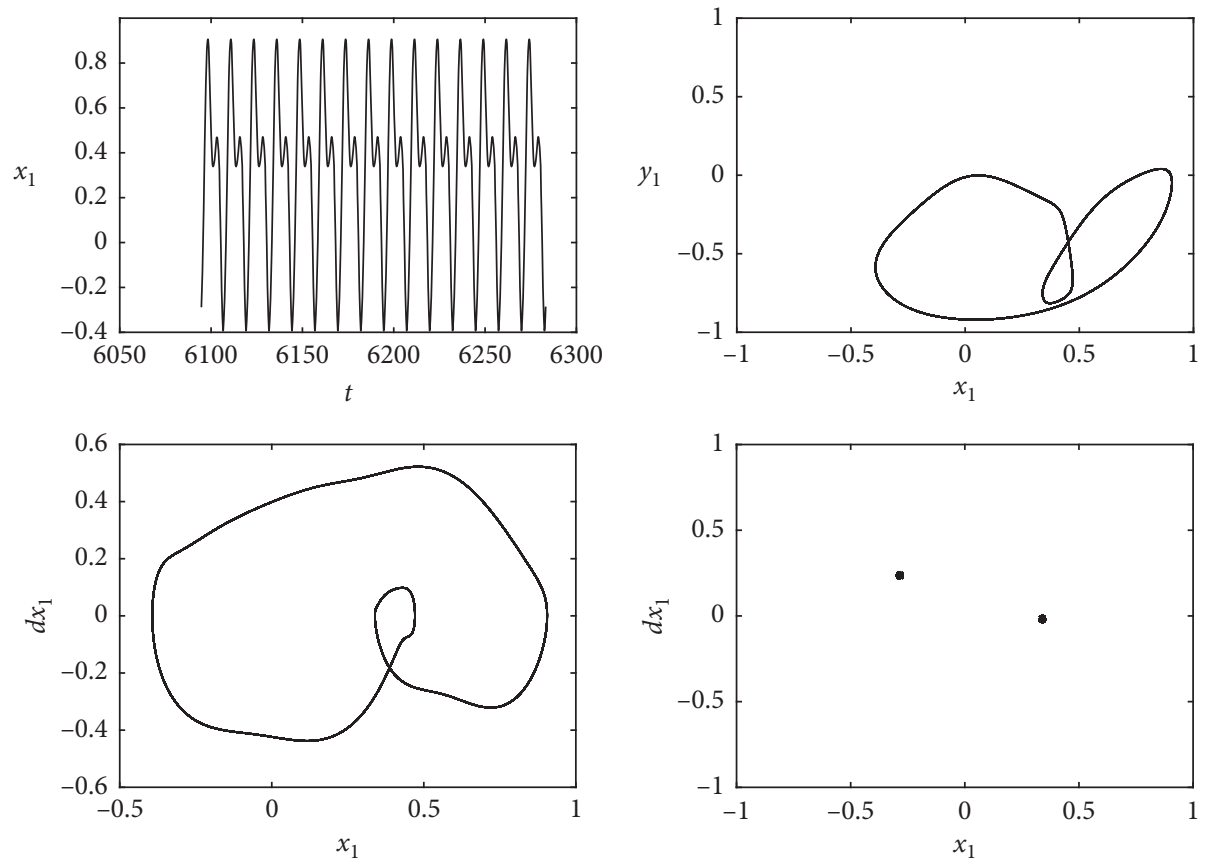

(a)
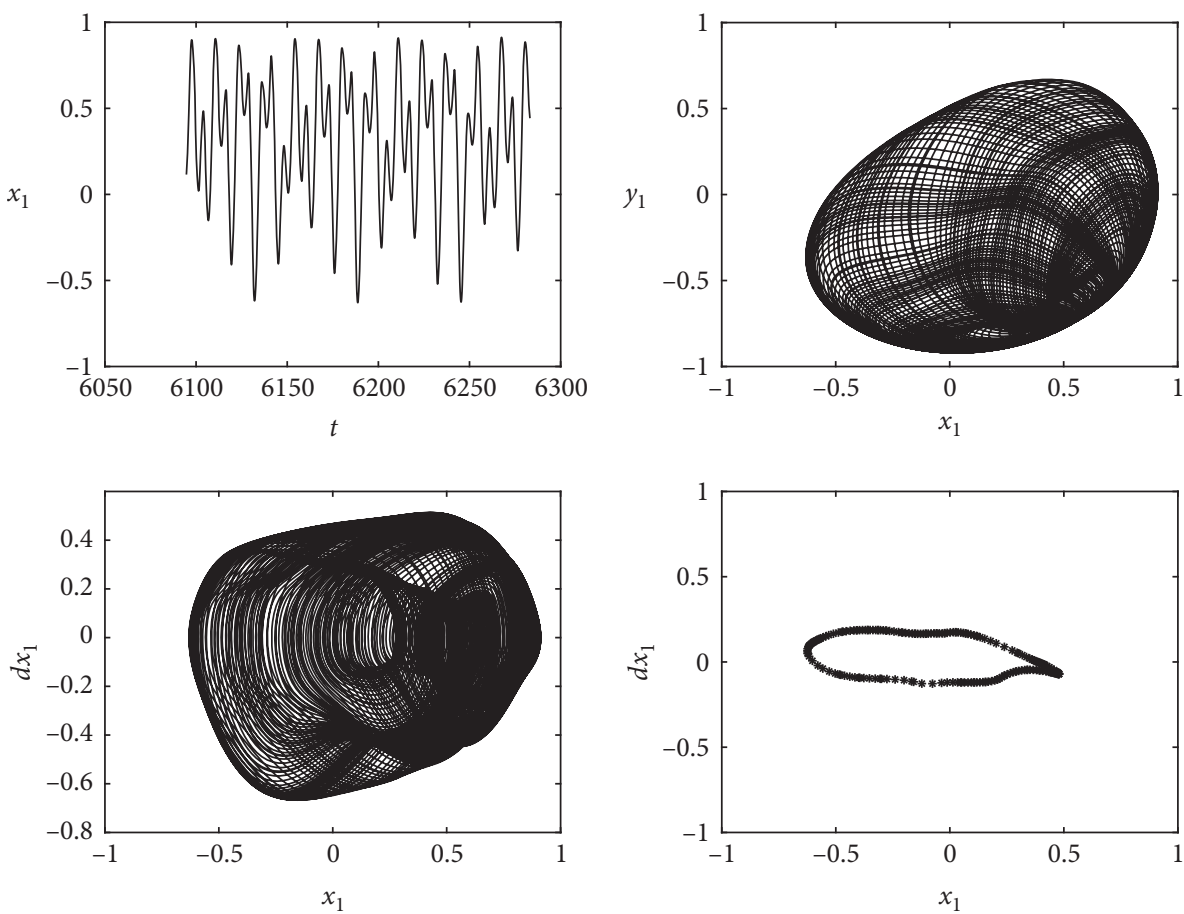

(b)

Figure 8: Continued. 

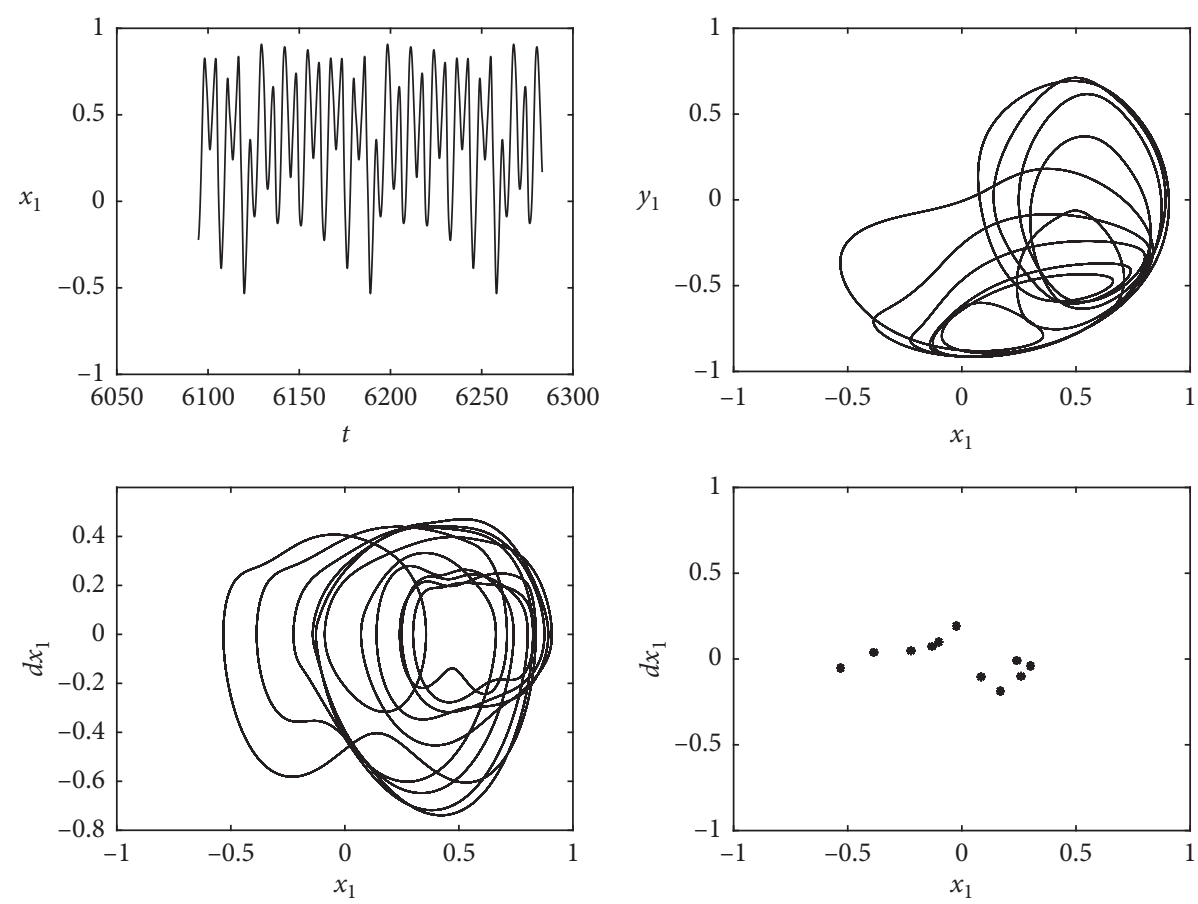

(c)
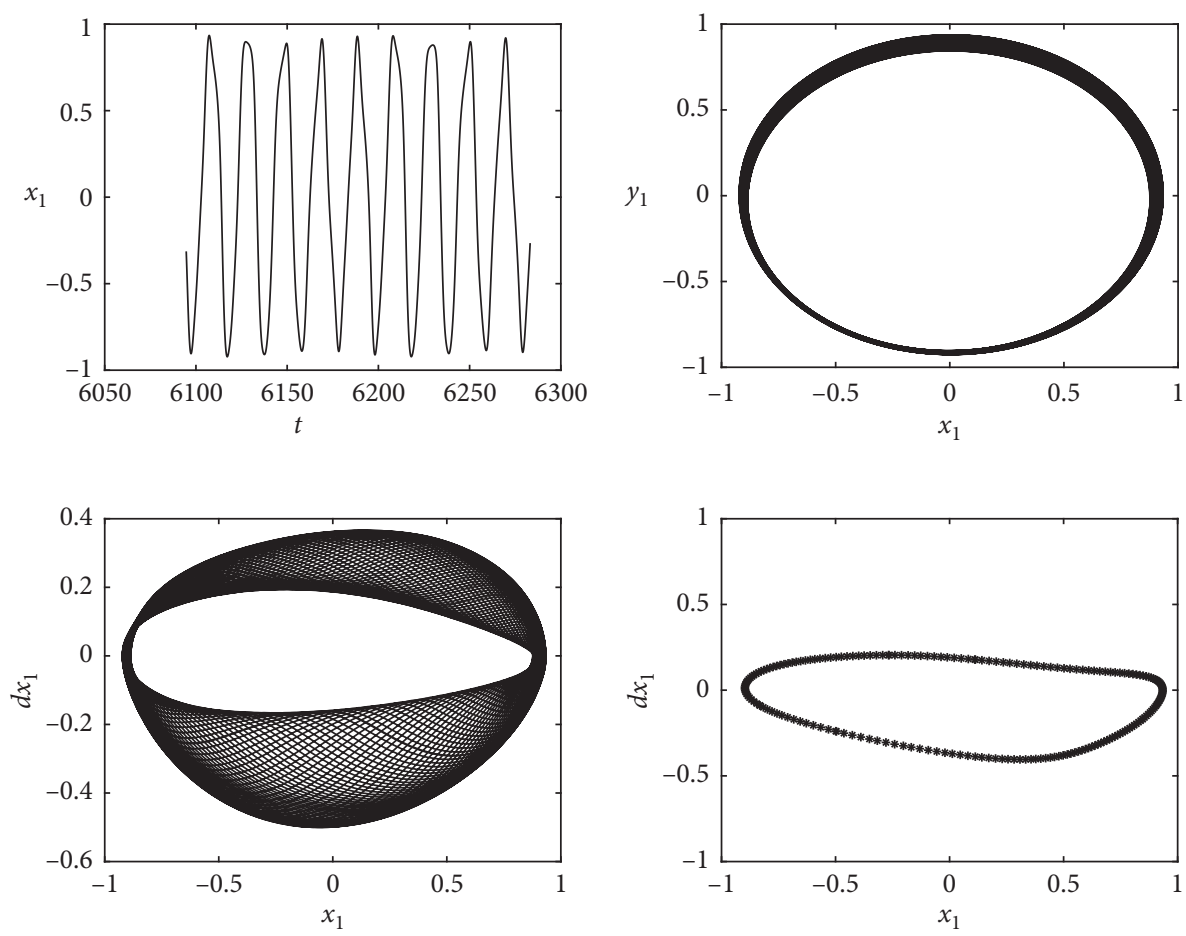

(d)

FiguRE 8: Response of the rotor system at different rotation speeds when $b_{1}=0.01 \mathrm{~mm}$ : (a) $\omega=575 \mathrm{rad} / \mathrm{s},(\mathrm{b}) \omega=725 \mathrm{rad} / \mathrm{s},(\mathrm{c}) \omega=825 \mathrm{rad} / \mathrm{s}$, and (d) $\omega=1900 \mathrm{rad} / \mathrm{s}$.

in the period 11 motion state. As the speed increases, the frictional force of the system begins to increase and gradually becomes the main factor affecting the motion state of the system.
When the rotational speed is greater than $900 \mathrm{rad} / \mathrm{s}$, the rotor system leaves the quasi-periodicity region through the reverse bifurcation process and becomes a quasi-periodicity one. When the rotational speed increases to $1525 \mathrm{rad} / \mathrm{s}$, the 
rotor system again branches into a wide range of quasiperiodic motion. Figure $8(\mathrm{~d})$ is the response of the system when $\omega=1900 \mathrm{rad} / \mathrm{s}$, where the time-domain waveform is regular, the axis trajectory is close to the limit cycle, and the Poincaré section is a closed curve. At this time, the system performs quasiperiodic motion.

Figure 9 shows the bifurcation response of the rotor system with an eccentric disc of $0.03 \mathrm{~mm}$. It can be seen P-1-P-2-quasi-periodicity-P-2-quasi-periodicity-P2-P-1-P-3-alternative cycle motion process. With the increase of the eccentricity of the left end of the disc, the system's quasiperiodic motion range is reduced and the cycle two movement appears in the range of 500-1000 rad/s. At the same time, a small range of nearly quasiperiodic motion range appears around $\omega=1000 \mathrm{rad} / \mathrm{s}$.

When the rotor rotates at a low speed, the system performs a stable quasiperiodic motion in the range of $200-525 \mathrm{rad} / \mathrm{s}$, and in the speed range $200-500 \mathrm{rad} / \mathrm{s}$, it is a cycle one movement. When the rotational speed exceeds $500 \mathrm{rad} / \mathrm{s}$, the system is affected by the whirl of the oil film. The double-cycle bifurcation becomes a quasi-periodicity second movement. As shown in Figure 10(a), the Poincaré section is the two isolated points.

With the increase of the rotational speed, the whirl of the oil film at the bearing begins to gradually intensify and finally the oil film destabilizes, and the system continues to branch into the quasiperiodic motion. The rubbing force during the system's quasi-periodicity movement is also increasing, but the film force is still the main factor affecting the stability of the system. Figure 10 (b) is the response of the system at a system speed of $600 \mathrm{rad} / \mathrm{s}$. There is a "clipping" phenomenon in the time history diagram. The Poincare section is a closed curve. At this time, the system performs quasiperiodic motion.

As the rubbing force increases, the effect of oil film whirl at the bearing is weakened, and the rotor system leaves the quasi-periodicity cycle and enters the cycle two movement with an interval of $650-925 \mathrm{rad} / \mathrm{s}$. As the rotational speed continues to increase, the rubbing force will continue to increase and gradually become the main factor affecting the operation of the system. The system will once again produce a bifurcation to enter the quasiperiodic motion state. Figure 10(c) shows the response of the system at a speed of $1100 \mathrm{rad} / \mathrm{s}$. The Poincaré section changes from a closed curve to two closed curves. It shows that the oil film whirl becomes oil film oscillation at the bearing, and the system is in quasiperiodicity movement.

The speed continues to increase, and the rotor system moves backwards into cycle three. When the speed exceeds $1550 \mathrm{rad} / \mathrm{s}$, the rotor system will enter the quasiperiodic motion, as shown in Figure 10(d). At this point, the trajectory of the axis is close to the limit cycle, and the Poincaré cross section is a closed curve. The rotor system performs quasiperiodic motion.

The response bifurcation diagram with the change of rotation speed is compared when the eccentricity is $0.01 \mathrm{~mm}, 0.03 \mathrm{~mm}$, and $0.05 \mathrm{~mm}$. It can be seen that when the rotation speed is low, the rotor system motion is not affected by the eccentricity, and it is a stable cycle one and a

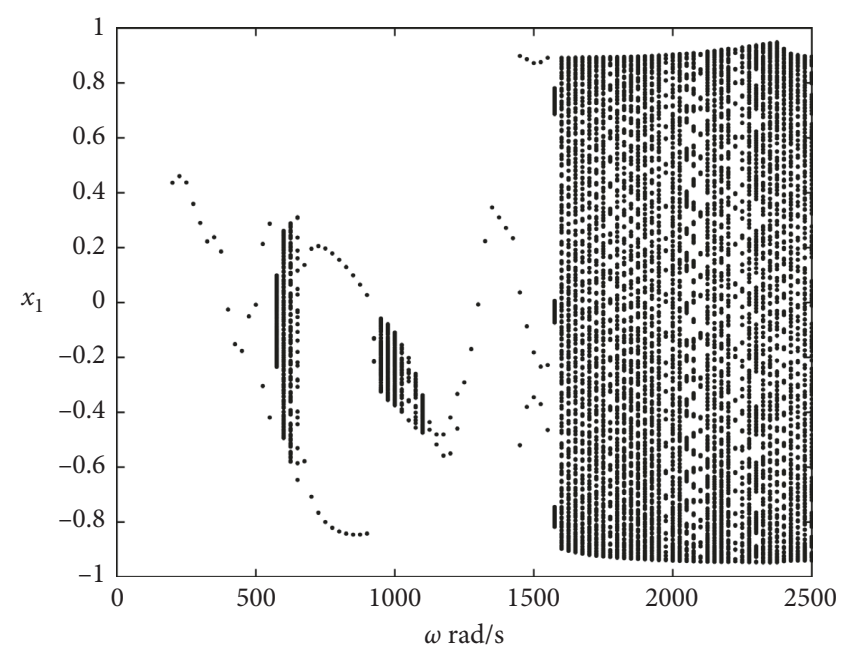

FIgURE 9: Bifurcation diagram of the rotor system in the $x_{1}$ direction when $b_{1}=0.03 \mathrm{~mm}$.

cycle two movement. The amplitude at the bearing is small, and there is a sharp corner. As the amount of eccentricity increases, the rotor system begins to appear in more abundant forms of movement. In the range of 500$1000 \mathrm{rad} / \mathrm{s}$, as the amount of eccentricity increases, the system's quasiperiodic motion interval decreases and the period two motion intervals appear. When $b_{1}=0.05 \mathrm{~mm}$, the two periods of this period evolve into chaos with two islands. In the range of more than $1000 \mathrm{rad} / \mathrm{s}$, the system's quasiperiodic motion area gradually increases. Three cycles of motion occur in the range of 1500-2000 rad/s when the eccentricity is $0.03 \mathrm{~mm}$ and $0.05 \mathrm{~mm}$. Under different eccentricity conditions, the rotor system is in quasiperiodic motion in the high-speed range. When the rotation speed is around $2500 \mathrm{rad} / \mathrm{s}$, the axial trajectory is close to the limit ring at the bearing.

4.3. Effect of Stator Stiffness on Response of Rotor System. According to the calculation formula of the rotor-impact frictional force model, it can be seen that the stator stiffness $k_{c}$ is one of the main factors affecting the size of the statortorsion force. In this section, by selecting different stator stiffness, the influence of the system on the response bifurcation diagram with changes in speed is studied. In this section, the eccentricity of the two discs is $0.05 \mathrm{~mm}$, and the other parameters remain unchanged.

Figure 11 shows the response bifurcation diagram of the rotor system with the change of the speed when the stator stiffness $k_{c}=3 \times 10^{7} \mathrm{~N} / \mathrm{m}$. It can be seen from the figure that the system has undergone the P-1-P-2-P-K-chaotic-P-K-P-2alternate cycle-P-3-alternate quasiperiodic motion process. When the rotor speed is low, the oil film force is the main factor affecting the stability of the system. When the system at a speed of $500 \mathrm{rad} / \mathrm{s}$, the system makes a quasiperiodic motion. As the rotation speed increases, the oil film is unstable and a whirl occurs, and the rotor system enters cycle two movement. Figure 12(a) shows the response of the system at $600 \mathrm{rad} / \mathrm{s}$. The axis trajectory is two nested rings. The Poincare section is two 

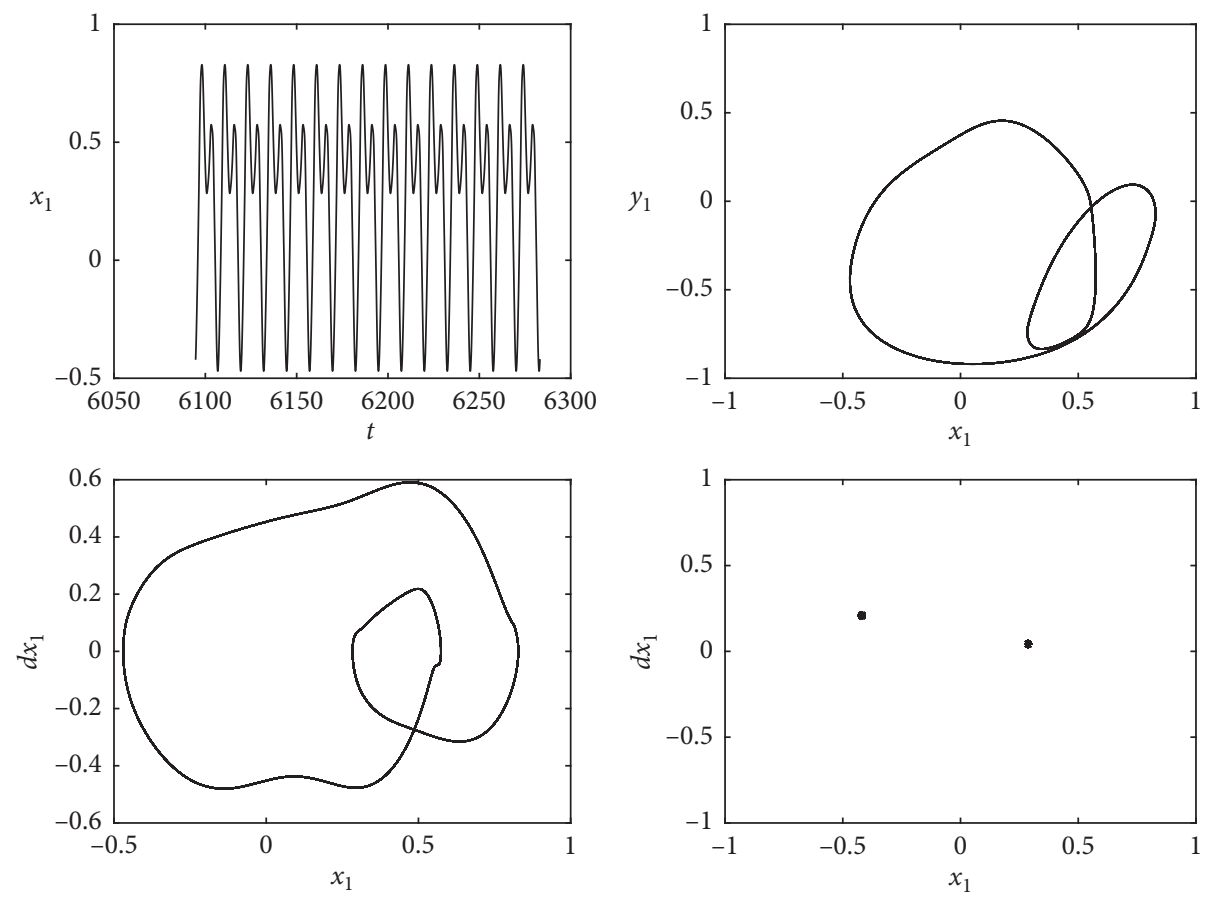

(a)
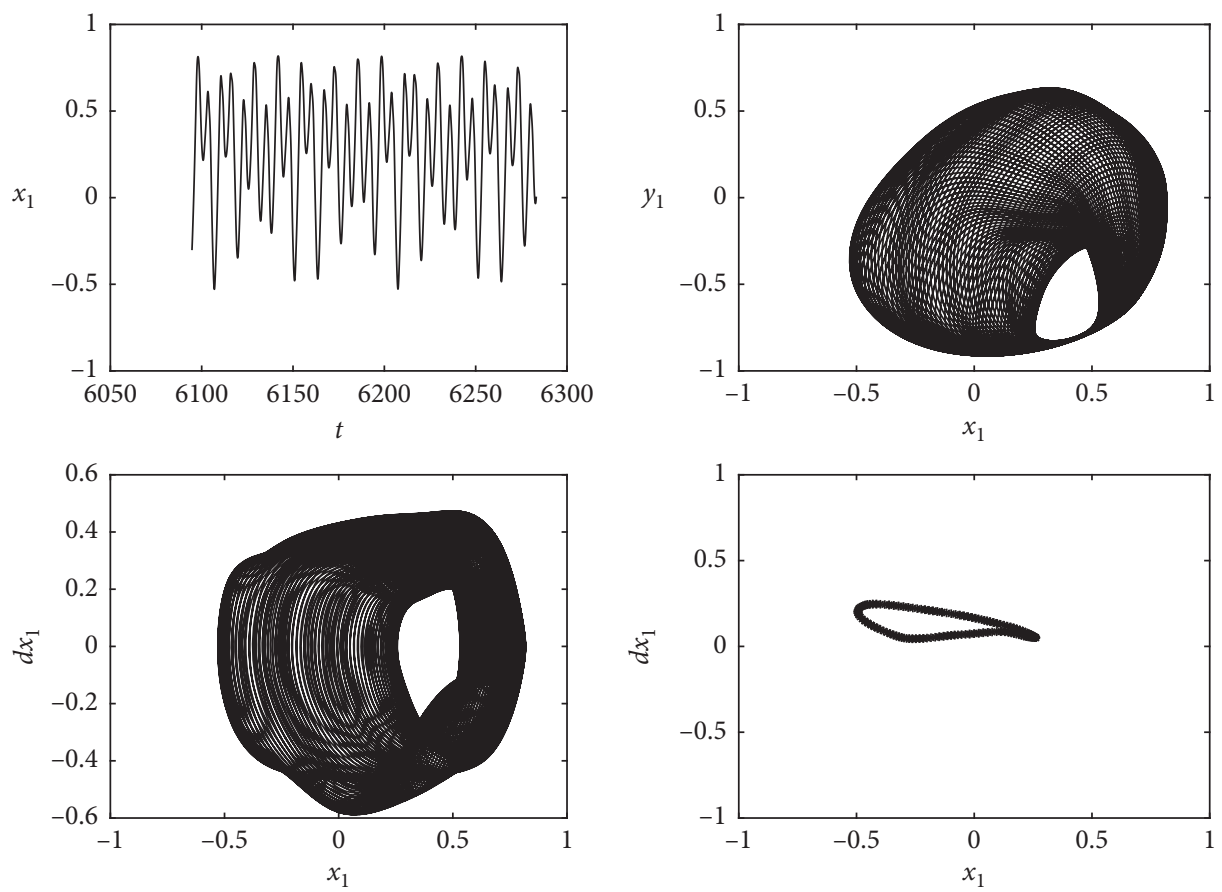

(b)

Figure 10: Continued. 

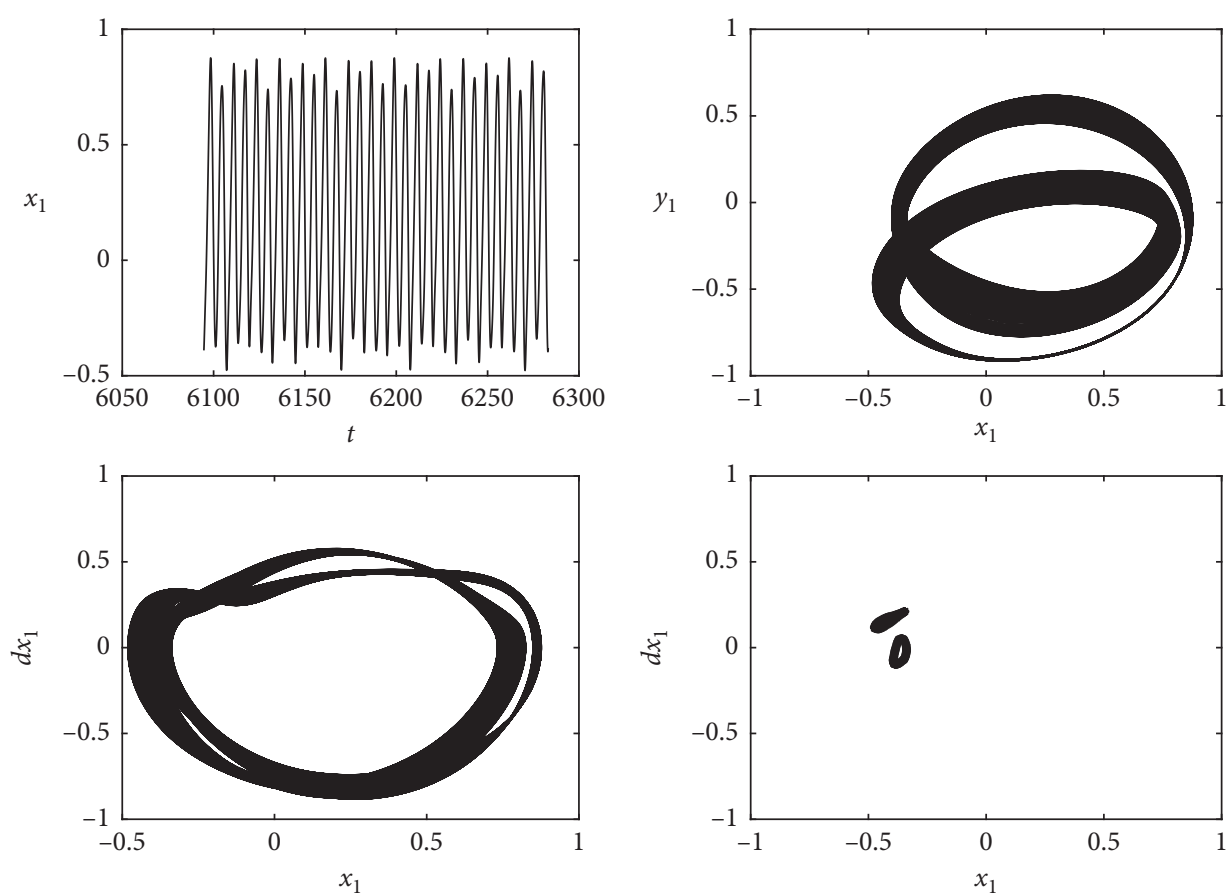

(c)
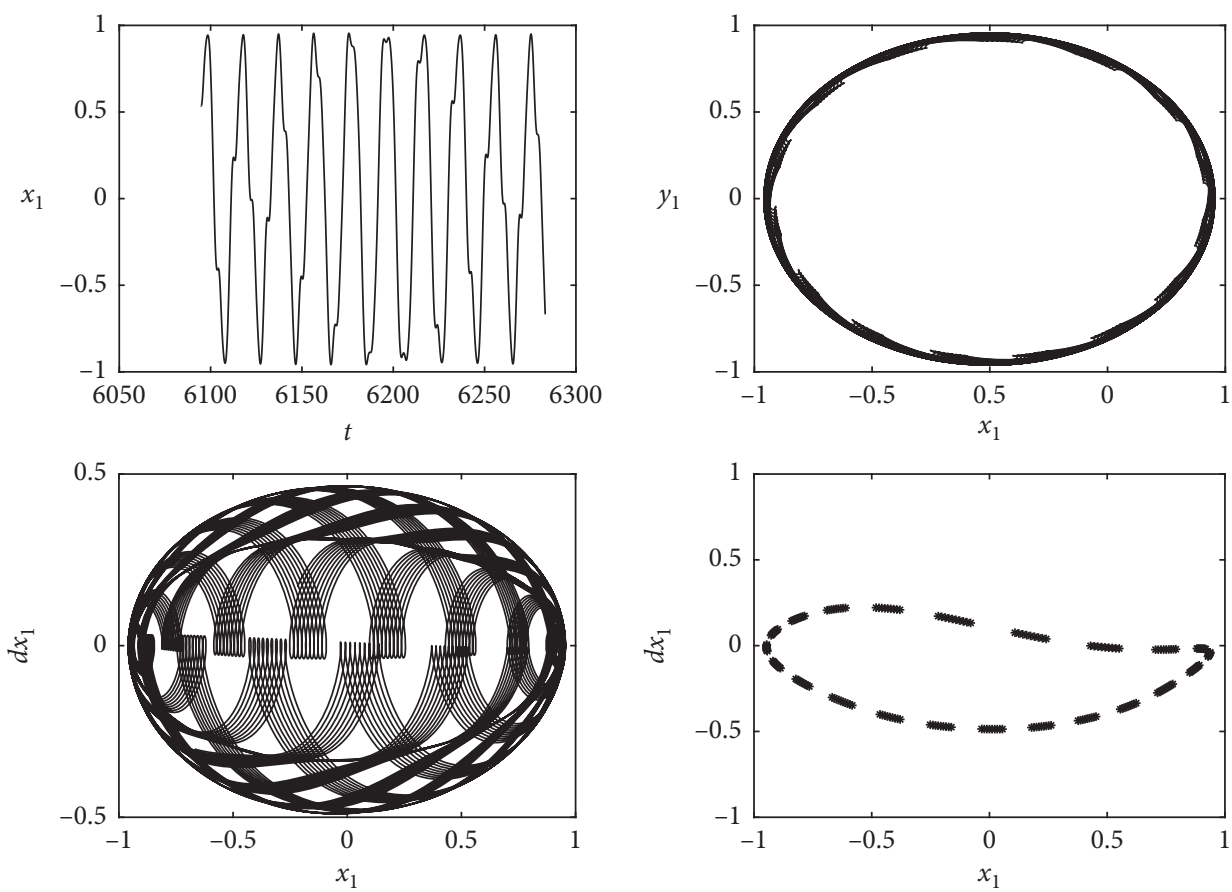

(d)

Figure 10: Response of the rotor system at different rotation speeds when $b_{1}=0.03 \mathrm{~mm}$ : (a) $\omega=550 \mathrm{rad} / \mathrm{s}$, (b) $\omega=600 \mathrm{rad} / \mathrm{s},(\mathrm{c}) \omega=1100 \mathrm{rad} / \mathrm{s}$, and (d) $\omega=2300 \mathrm{rad} / \mathrm{s}$. 


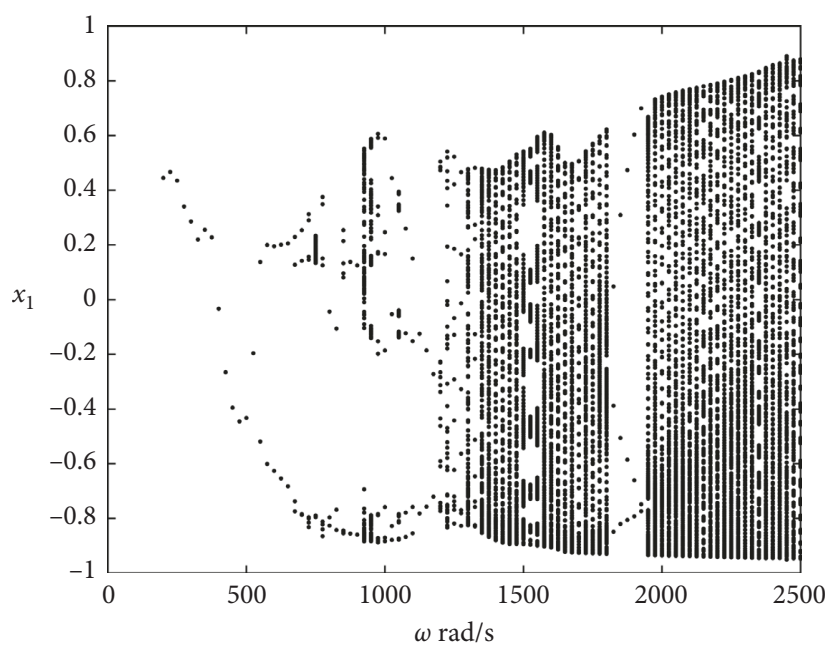

Figure 11: Bifurcation diagram of the rotor system in the $x_{1}$ direction when $k_{c}=3 \times 10^{7} \mathrm{~N} / \mathrm{m}$.

isolated points. This shows that the system has a doubled period of time for the cycle two movements.

With the intensification of the oil film vortex, the system continues to branch and enters the P-K cycle. When the system at a speed of $775 \mathrm{rad} / \mathrm{s}$, the system makes a period 7 movement. When the rotor speed reaches $925 \mathrm{rad} / \mathrm{s}$, the system oil film is in chaos. Figure 12(b) is the response of the system when $\omega=925 \mathrm{rad} / \mathrm{s}$, the time-domain plot is an irregular waveform, the axis trajectory is a misaligned plot, and the Poincaré cross section is a plot of two islands formed by a point. At this point, the system's movement is in chaos.

Due to the presence of eccentricity in the system, there is always an imbalance force in the rotor system. According to the unbalanced force term in the system dynamics differential equation group, it can be known that when the rotation speed is low, the unbalanced force is small and the system stability is less affected. At this time, the film force is the main factor affecting the stability of the system. With the increase of the rotational speed, the unbalanced force in the system will gradually increase and gradually become the main factor affecting the stability of the system. When the rotation speed exceeds $1050 \mathrm{rad} / \mathrm{s}$, the rotor system goes backwards into the cycle two movement. Figure 12(c) shows the response of the system at $1525 \mathrm{rad} / \mathrm{s}$. There is a "clipping" phenomenon in the time history diagram. The Poincare section is a closed curve, and the system performs quasiperiodic motion. As the speed increases further, the system enters the cycle three movement. As shown in Figure 12(d), the trajectory of the axis is three intersecting rings, and the Poincaré section is three isolated points. When the speed exceeds $1925 \mathrm{rad} / \mathrm{s}$, the system again enters quasiperiodic motion. Figure 12(e) is the response of the system at a speed of $2500 \mathrm{rad} / \mathrm{s}$. The observation chart shows that the phenomenon of "beat vibration" appears in the time-domain diagram. The Poincaré section is a closed curve, and the axis trajectory is closer to the limit cycle at the bearing. At this time, the rotor system makes a quasiperiodic motion.
Figure 13 shows the response bifurcation of the rotor system with $k_{c}=5 \times 10^{7} \mathrm{~N} / \mathrm{m}$. It can be seen from the figure that the system is within the $200-2500 \mathrm{rad} / \mathrm{s}$ speed range. It has experienced P-1-P-2-P-4-chaos-P-2-chaos-P-2quasi-periodicity-P-3-quasiperiodic motion.

The system performs a stable quasiperiodic motion at low speeds with an interval of $200-675 \mathrm{rad} / \mathrm{s}$. When the rotation speed is less than $525 \mathrm{rad} / \mathrm{s}$, the system is in a cycle one movement. When the rotation speed exceeds $525 \mathrm{rpm}$, the system will cycle twice as much as the whirl of the oil film. As shown in Figure 14(a), the time-domain diagram is a regular waveform. The axis of the axis is two nested rings. The Poincaré cross section is two isolated points. At this time, the system performs quasi-periodicity two motion at this rotational speed.

The oil film vortex aggravating system continues to occur twice the period into the chaotic movement. As shown in Figure 14(b), the time-domain graph is an irregular waveform, the axis trajectory is an infinite number of noncoincident curves, and the Poincare section is a cloud-like irregular point. From this, it can be judged that the rotor is in a chaotic state at this rotational speed.

With the further increase of the rotational speed, the unbalanced force due to eccentricity in the system becomes larger. When the rotor speed exceeds $925 \mathrm{rad} / \mathrm{s}$, the system under the action of the unbalanced force goes through the backward bifurcation and enters the cycle two movement. In this process, the system instability caused by the oil film force is gradually offset, and the system movement is gradually stabilized. When the rotational speed increases to $1100 \mathrm{rad} / \mathrm{s}$, the system enters a short chaotic motion range $1050-1060 \mathrm{rad} / \mathrm{s}$ and then goes backwards again into cycle two.

When the speed exceeds $1200 \mathrm{rad} / \mathrm{s}$, the system goes into quasiperiodic motion. Figure 14(c) shows the response of the system at a speed of $1500 \mathrm{rad} / \mathrm{s}$. The Poincaré section is a closed curve. When the speed exceeds $1775 \mathrm{rad} / \mathrm{s}$, the system enters cycle three and the range is $1800-1950 \mathrm{rad} / \mathrm{s}$. As shown in Figure 14(d), the three axes 

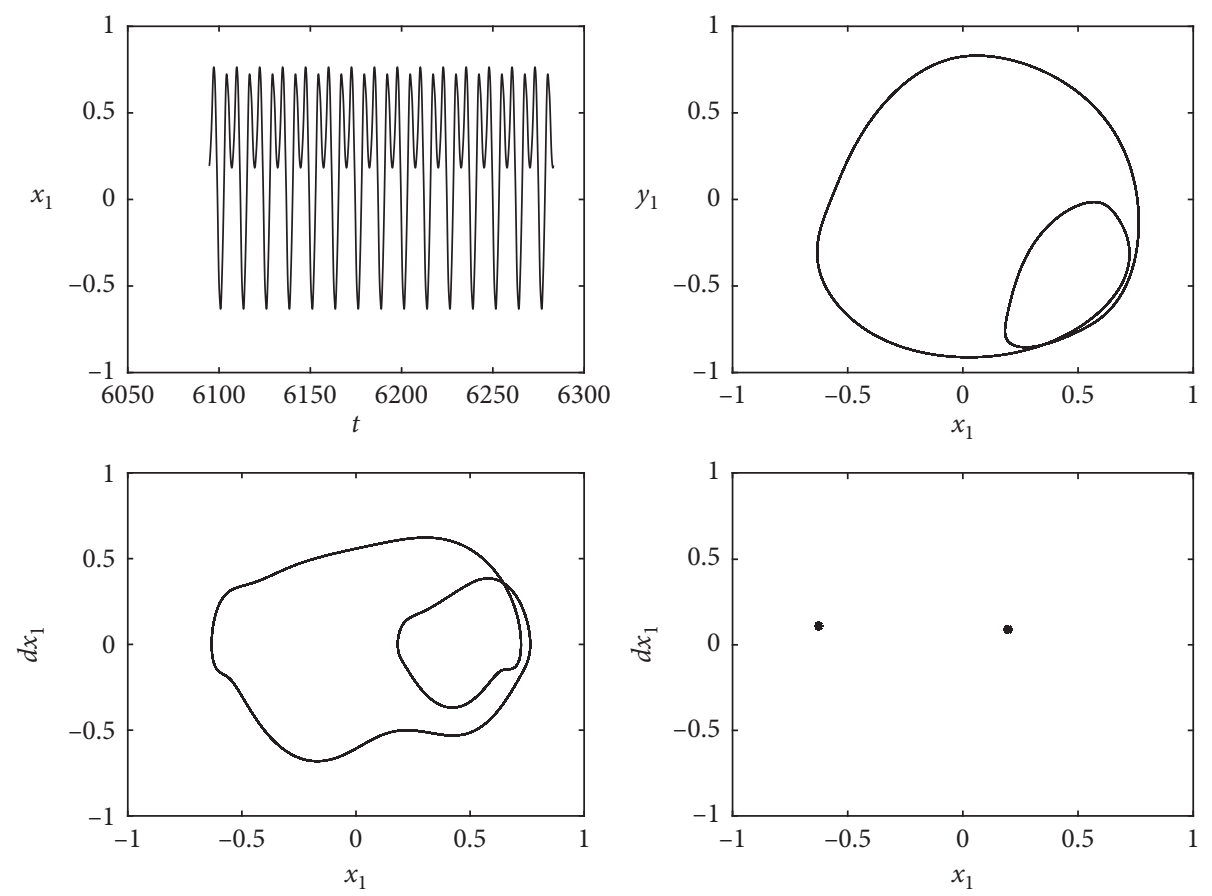

(a)
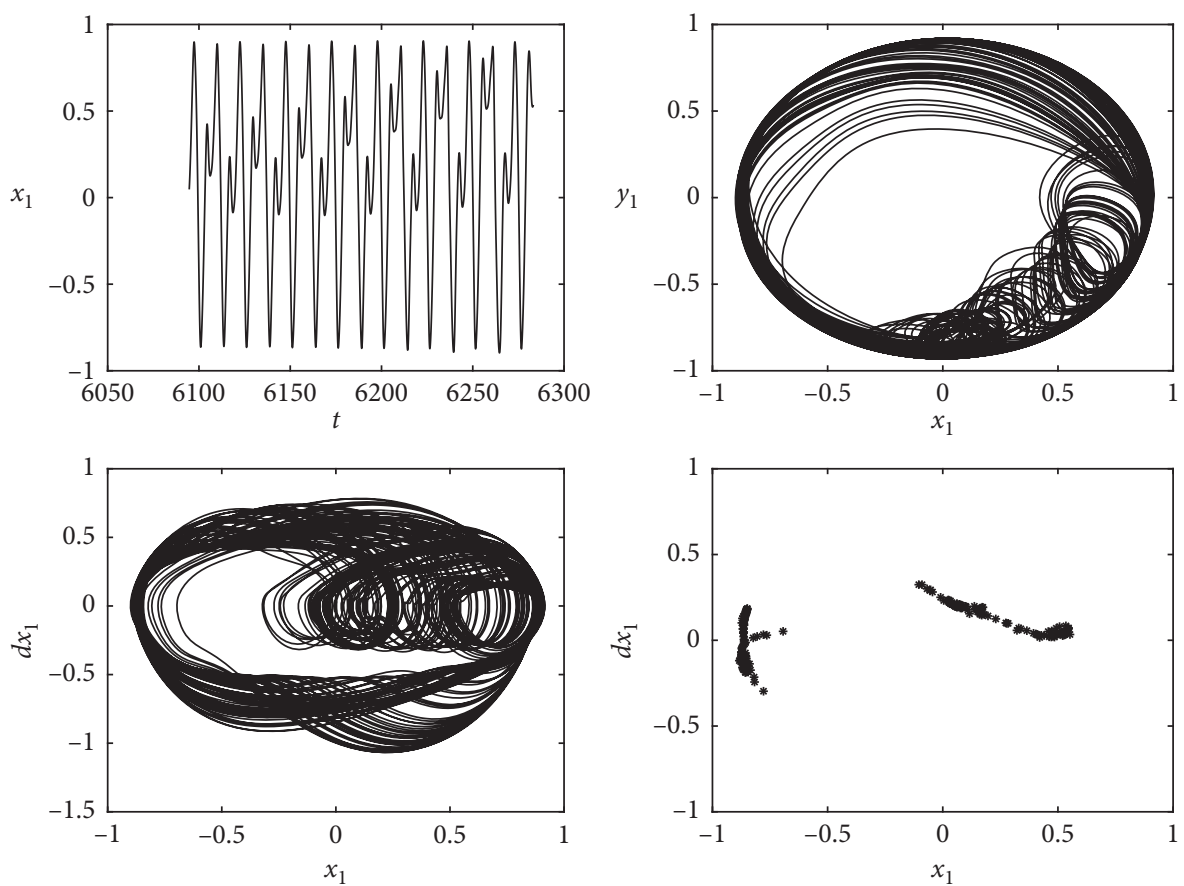

(b)

Figure 12: Continued. 

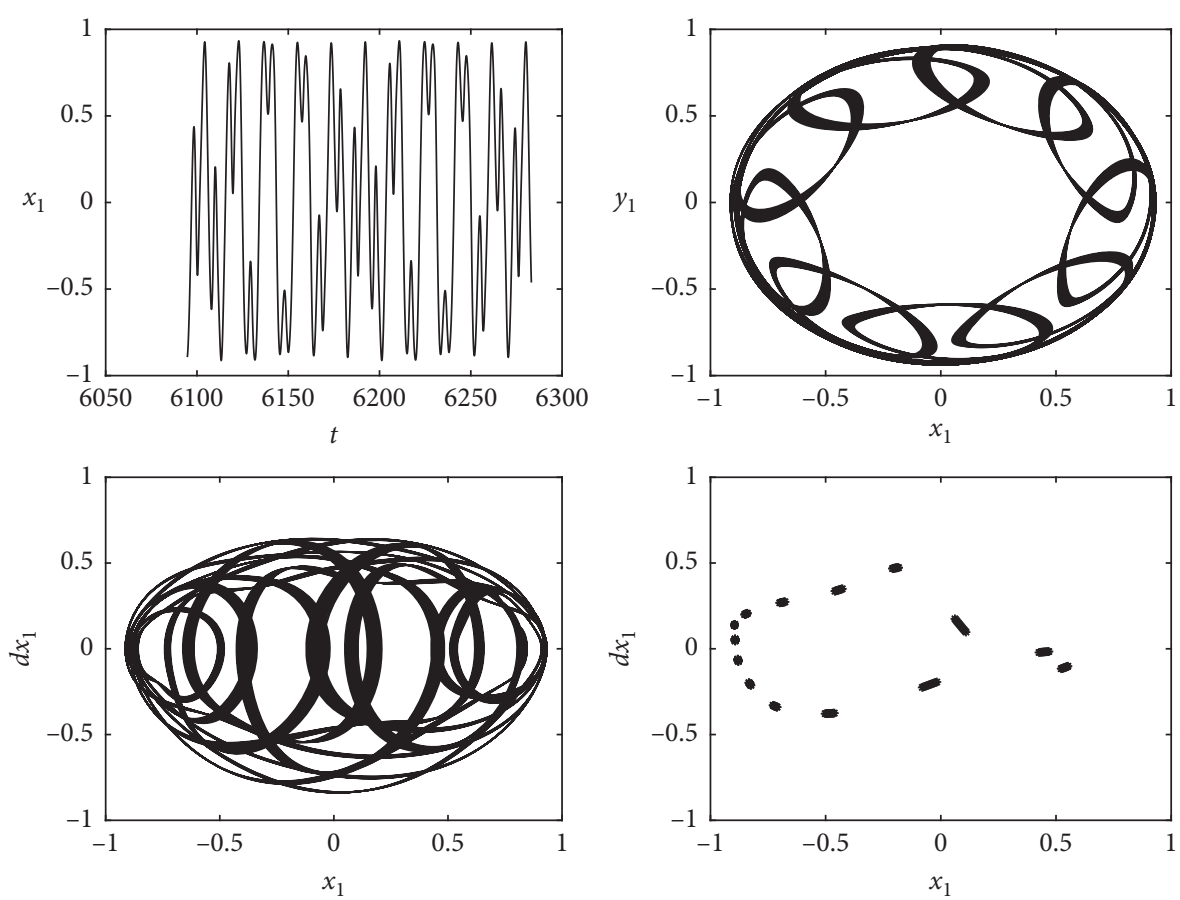

(c)
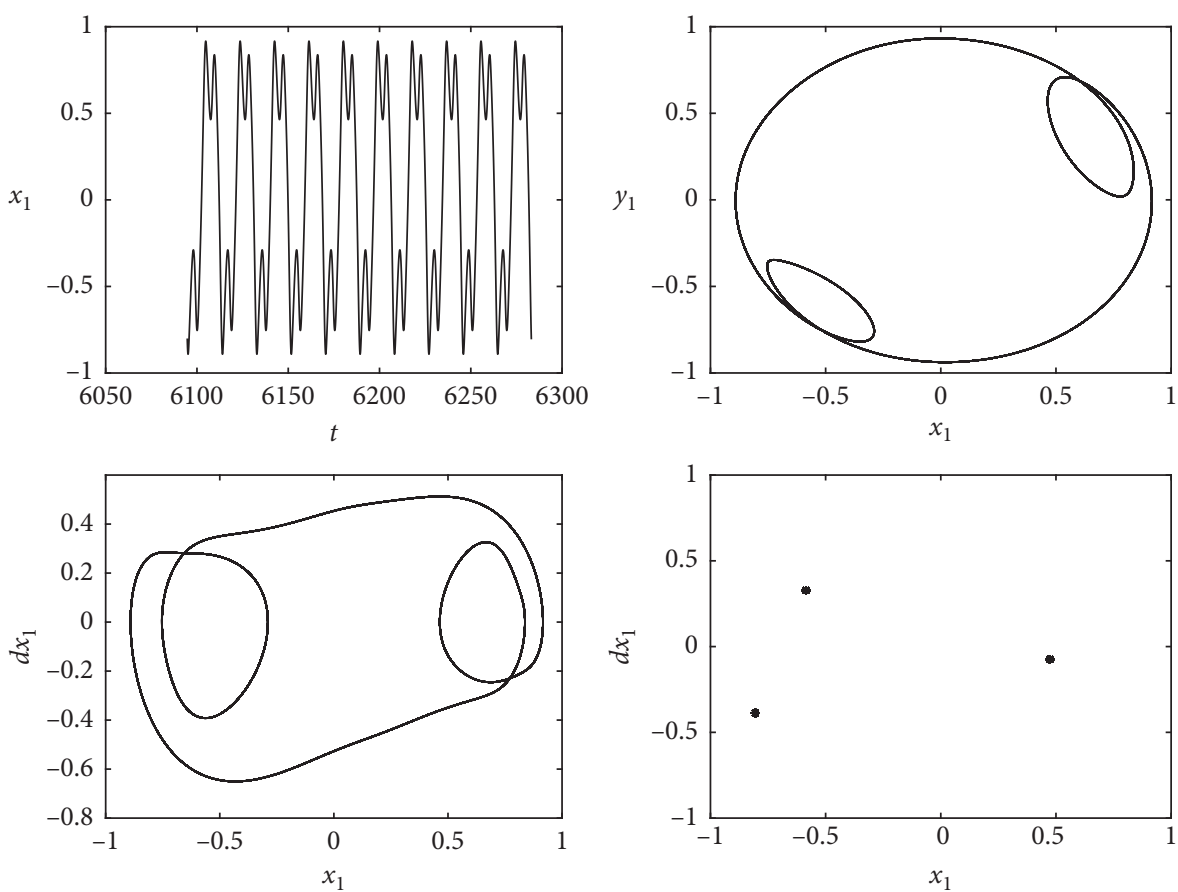

(d)

Figure 12: Continued. 

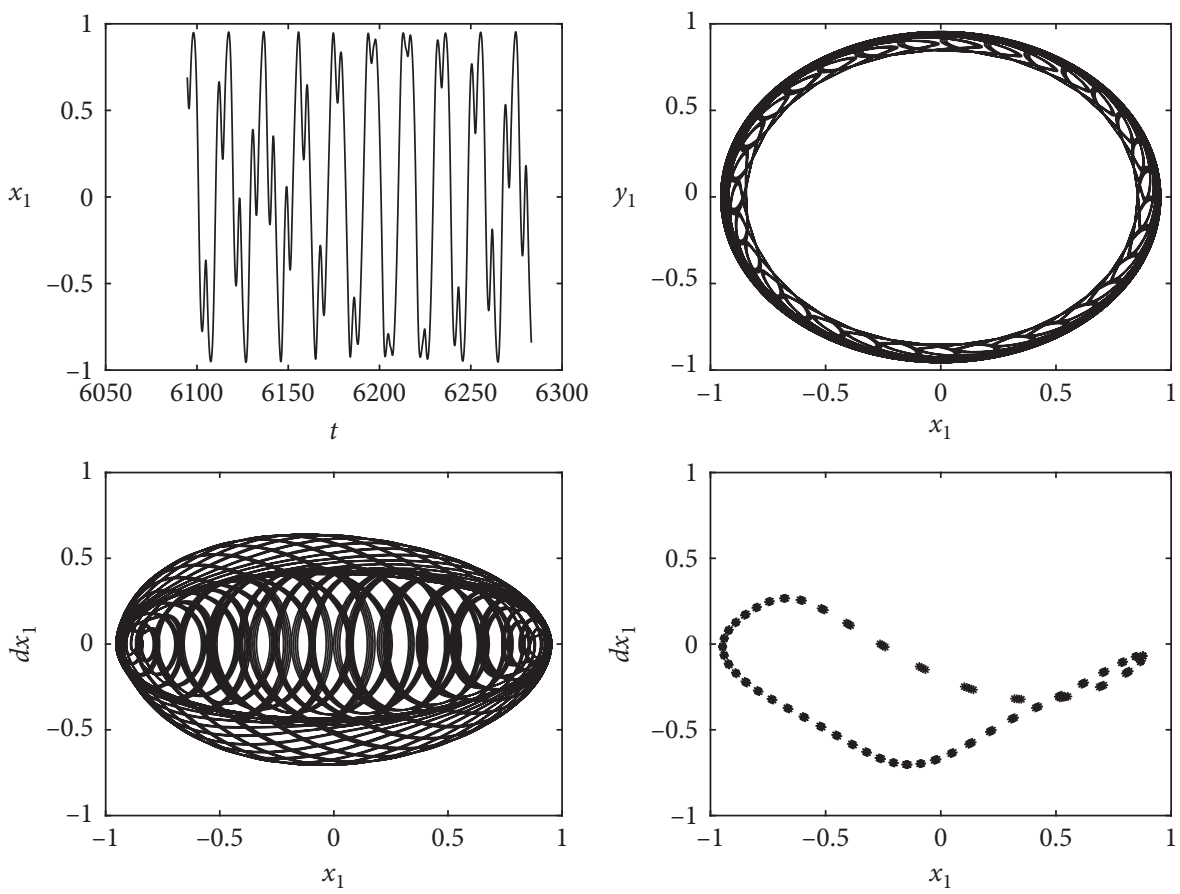

(e)

FIGURE 12: Response of the rotor system at different rotation speeds when $k_{c}=3 \times 10^{7} \mathrm{~N} / \mathrm{m}$ : (a) $\omega=600 \mathrm{rad} / \mathrm{s}$, (b) $\omega=925 \mathrm{rad} / \mathrm{s}$, (c) $\omega=1525 \mathrm{rad} / \mathrm{s}$, (d) $\omega=1875 \mathrm{rad} / \mathrm{s}$, and (e) $\omega=2500 \mathrm{rad} / \mathrm{s}$.

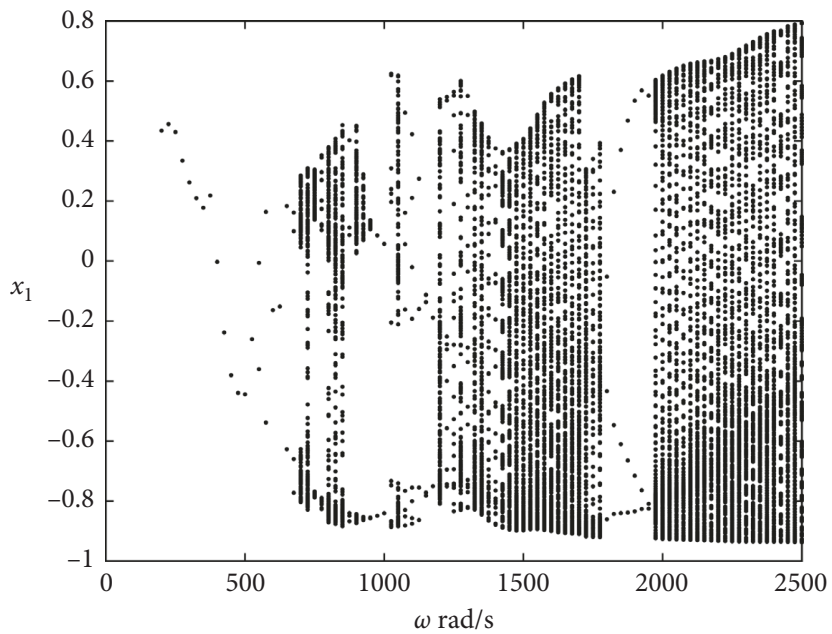

FIGURE 13: Bifurcation diagram of the rotor system in the $x_{1}$ direction when $k_{c}=5 \times 10^{7} \mathrm{~N} / \mathrm{m}$.

of the axis trajectory are nested, and the Poincaré section is three isolated points. The system performs quasi-periodicity three movements.

Figure 14(e) plots the response of the rotor system at $2400 \mathrm{rad} / \mathrm{s}$. In the time-domain diagram, there is a "clipping" phenomenon and there is a "beat vibration" phenomenon. The axis trajectory is close to the limit circle at the bearing. The Poincaré section is a closed curve, and the system performs quasiperiodic motion.

Comparing the response of the rotor system with different stator stiffness at $k_{c}$, we can see that the relative chaos in the speed range 500-1000 rad/s decreases with $k_{c}=3 \times 10^{7}$. When $k_{c}=5 \times 10^{7} \mathrm{~N} / \mathrm{m}$, the chaotic region increases again in this speed range, which means that increasing the stator stiffness can restrain the chaos vibration caused by oil film oscillation in the system to some extent. However, when the stiffness exceeds a certain value, chaotic regions will reappear. In the high-speed range, the system response is not affected, and the rotor system is in quasiperiodic motion. During this period, there is also a small range of cycle three movements, and there is no significant change in the corresponding range. 

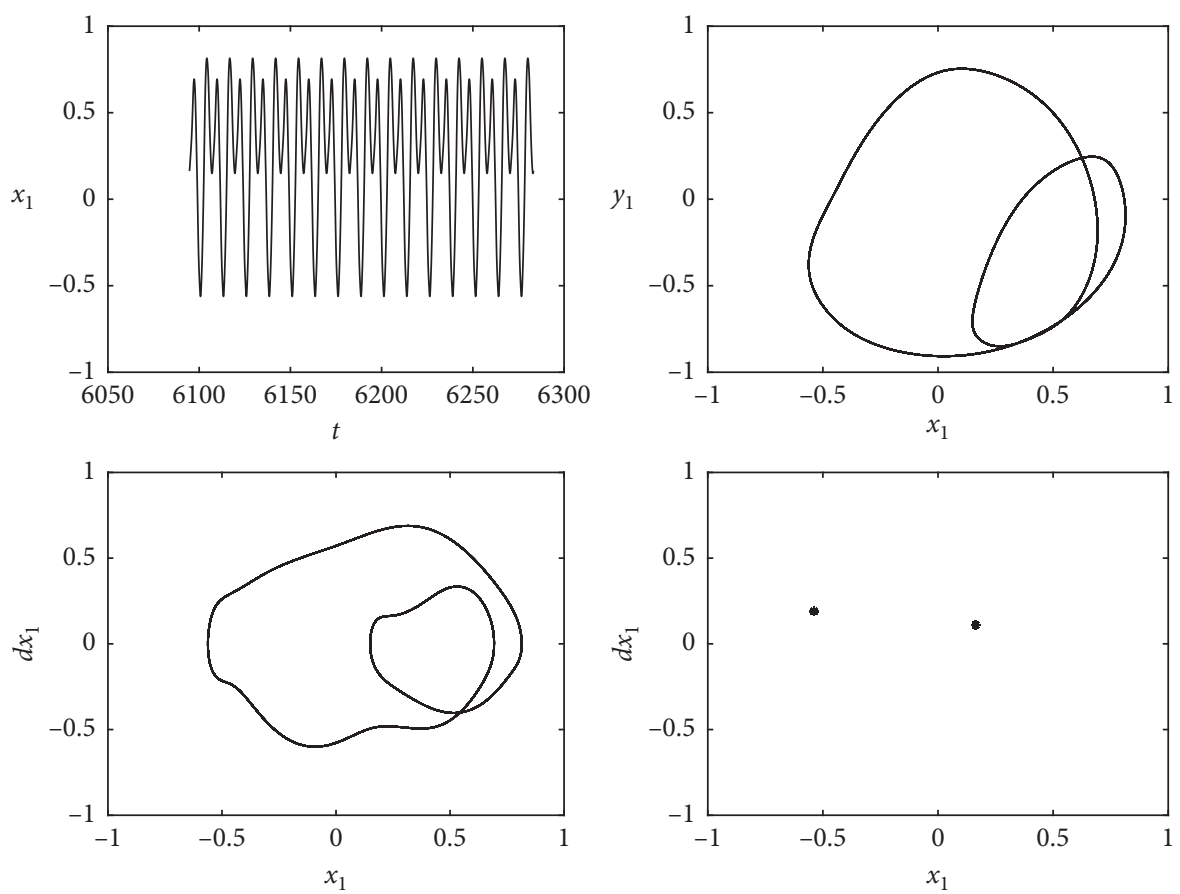

(a)
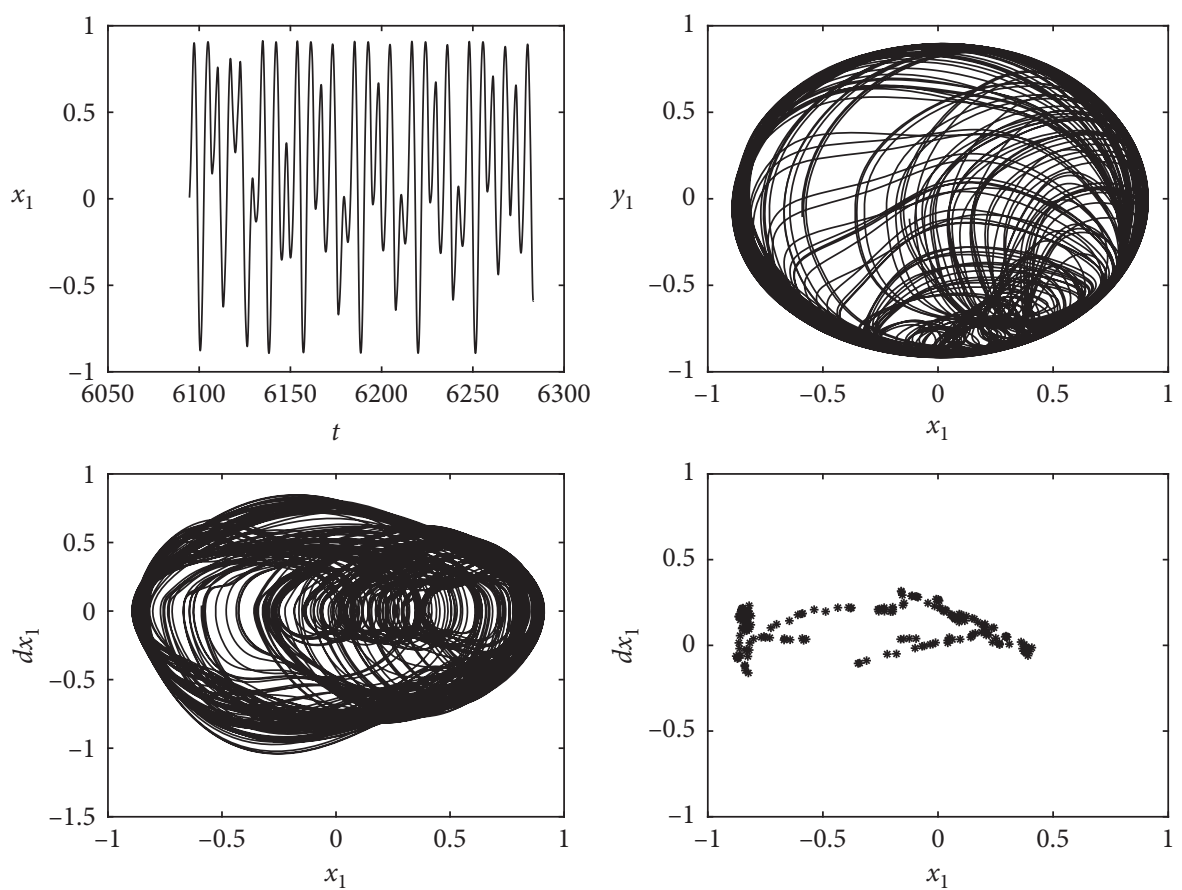

(b)

Figure 14: Continued. 

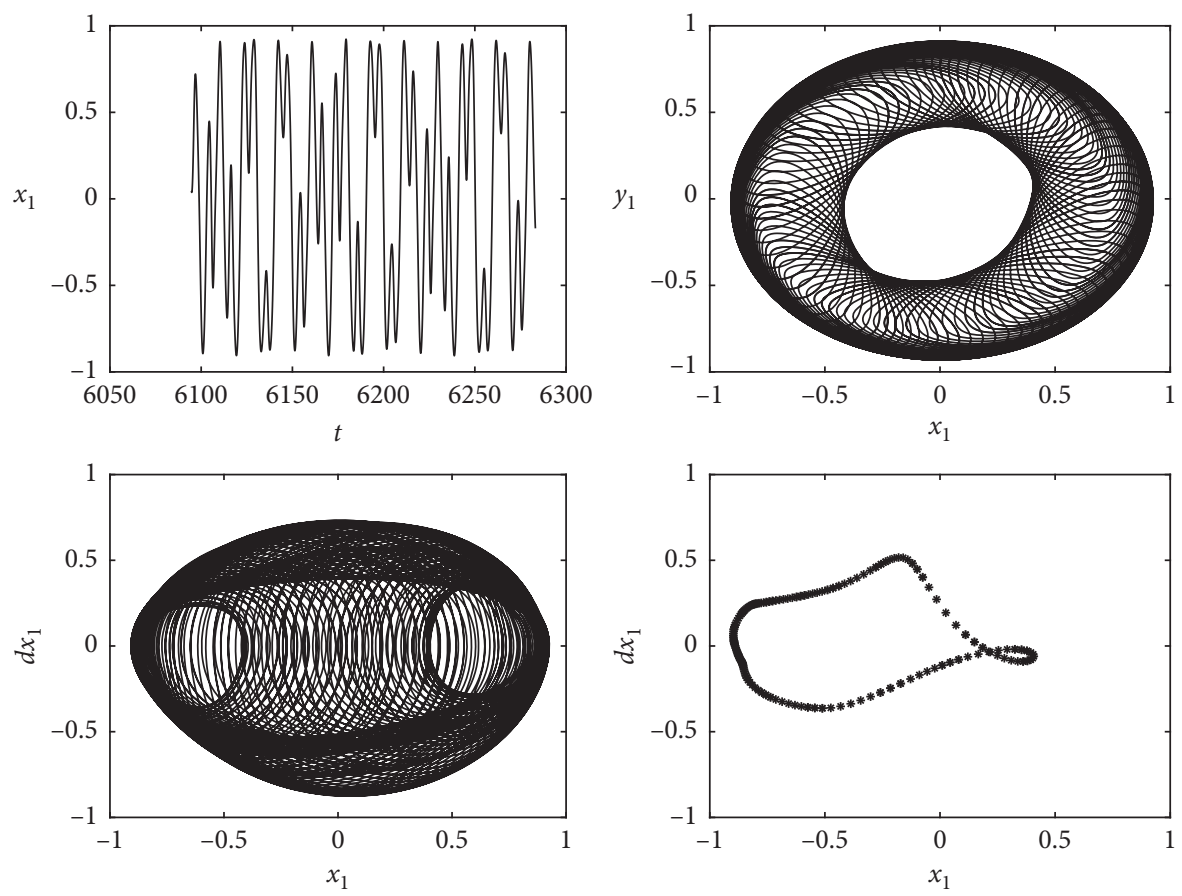

(c)
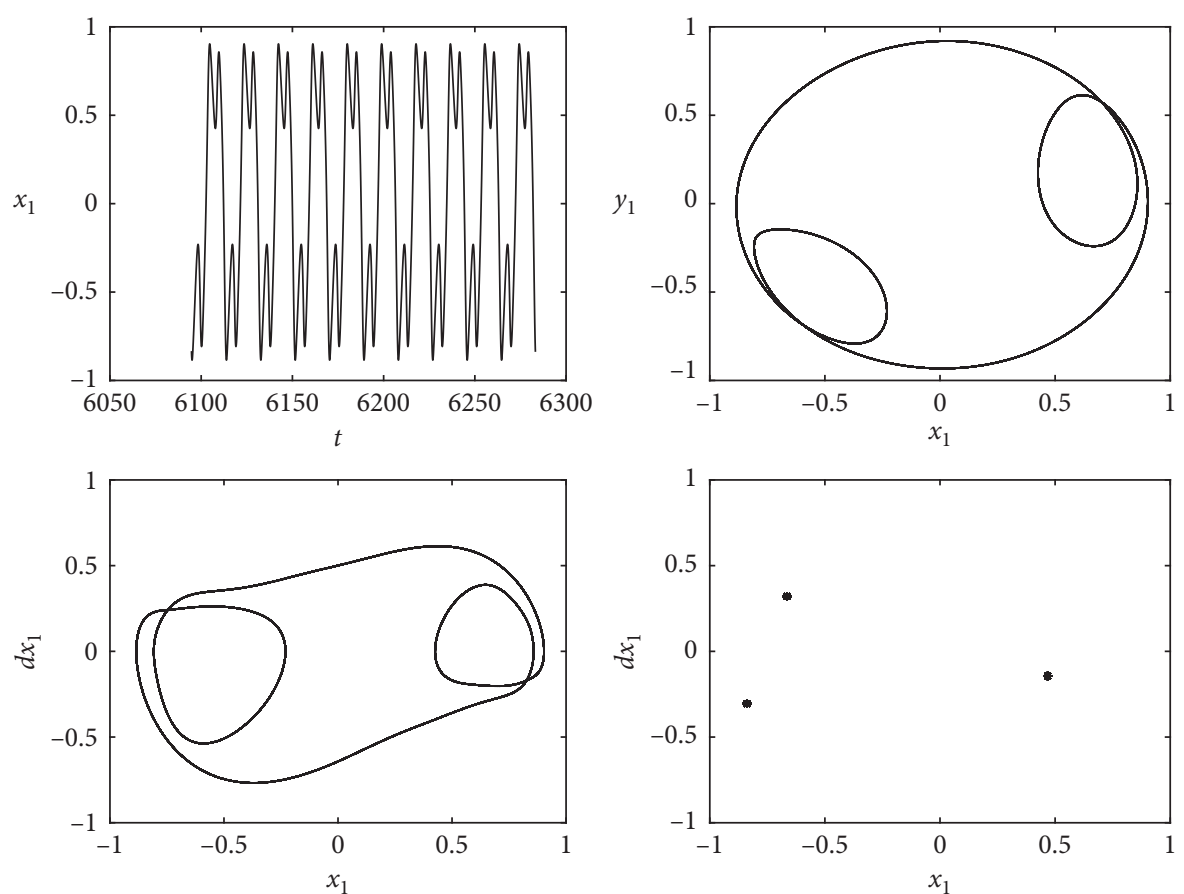

(d)

Figure 14: Continued. 

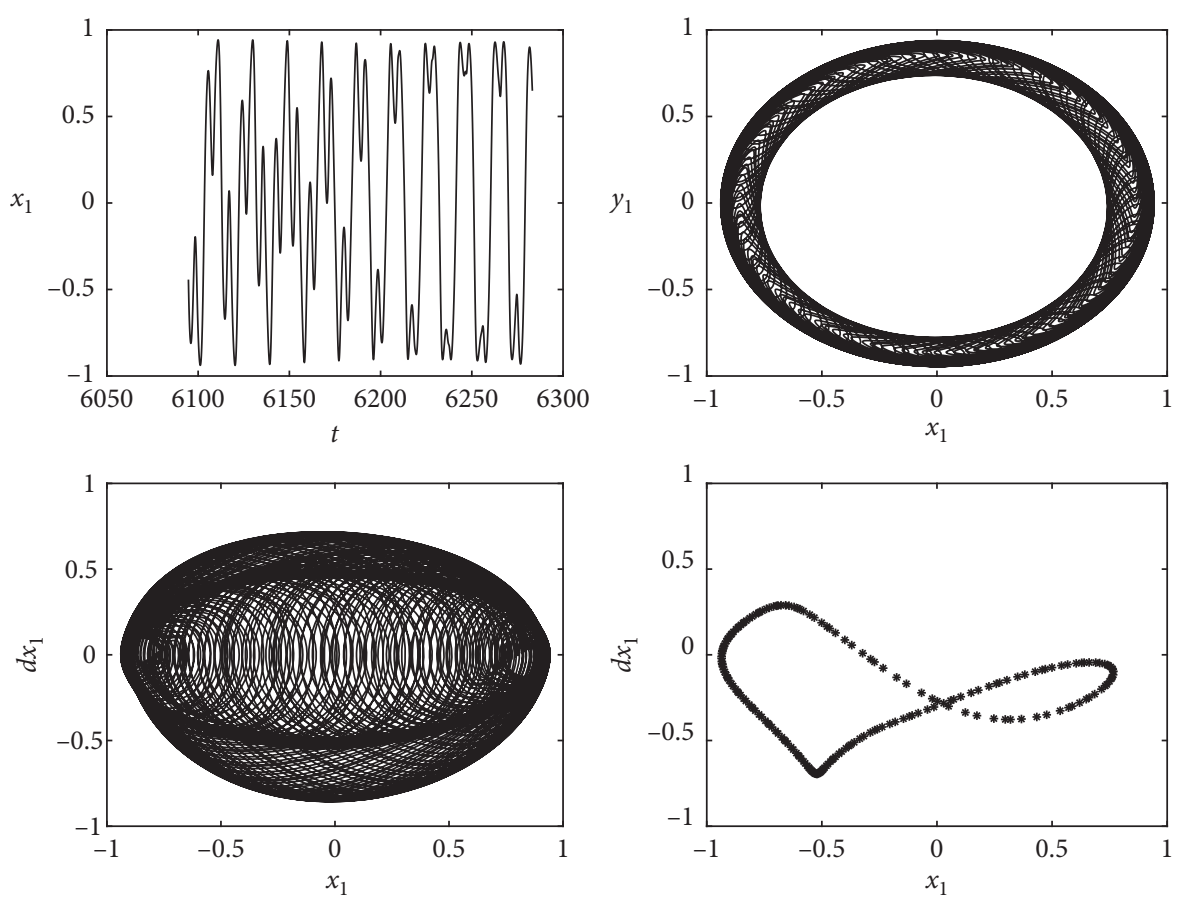

(e)

Figure 14: Response of the rotor system at different rotation speeds when $k_{c}=5 \times 10^{7} \mathrm{~N} / \mathrm{m}$ : (a) $\omega=575 \mathrm{rad} / \mathrm{s},(\mathrm{b}) \omega=825 \mathrm{rad} / \mathrm{s},(\mathrm{c}) \omega=1500 \mathrm{rad} / \mathrm{s}$, (d) $\omega=1875 \mathrm{rad} / \mathrm{s}$, and (e) $\omega=2400 \mathrm{rad} / \mathrm{s}$.

\section{Conclusions}

In this paper, the dynamic model of the double-disc rotorbearing system with rubbing fault is established. The numerical integration method is combined with MATLAB to solve the dynamic differential equation of the system. The effects of the speed of the rotor, the disc eccentricity, and the stator stiffness on the response of the rotor-bearing system are analyzed. The following conclusions are obtained:

(a) The rotor system moves periodically in the lowspeed range $200-767 \mathrm{rad} / \mathrm{s}$. As the speed of the rotor continues to increase, the influence of the oil film force begins to further increase. When the speed is greater than $767 \mathrm{rad} / \mathrm{s}$, the system loses stability and performs chaotic motion. When the speed is $\omega=984 \mathrm{rad} / \mathrm{s}$, the chaotic interval changes to the period two motion. After a short period of four intervals (1107-1146) rad/s, the system directly becomes an almost periodic motion and evolves into a periodic three motion at $\omega=1744 \mathrm{rad} / \mathrm{s}$. Finally, when $\omega=1855 \mathrm{rad} / \mathrm{s}$, the system enters a wide range of almost periodic motion intervals. The whole process of the rotor system enters chaos by doublecycle bifurcation. When the rotor speed is high, the system is dominated by almost periodic motion.

(b) The rotor system is sensitive to changes in the eccentricity of the disc. When the speed is low, the motion of the system is not affected by the amount of eccentricity, and it is a stable period one and two periods of motion. As the amount of eccentricity increases, the system begins to appear in more abundant forms of motion. In the range of 500-1000 $\mathrm{rad} / \mathrm{s}$, as the eccentricity increases, the system's periodic motion interval decreases and the period two motion interval appears. When $b_{1}=0.05 \mathrm{~mm}$, the second interval of the cycle evolves into chaotic motion with two islands. In the range of more than $1000 \mathrm{rad} / \mathrm{s}$, the system's quasiperiodic motion area gradually increases. Three cycles of motion occur in the range of $1500-2000 \mathrm{rad} / \mathrm{s}$ when the eccentricity is $0.03 \mathrm{~mm}$ and $0.05 \mathrm{~mm}$.

(c) The increase of stator stiffness in the rotor system can suppress the chaotic vibration caused by the oil film oscillation in the system to some extent. However, when the stiffness exceeds a certain value, the chaotic area will appear again. In the interval of more than $1500 \mathrm{rad} / \mathrm{s}$, the change of stator stiffness has little effect on the system response, and the rotor system is in the almost periodic motion state. During this period, there is also a small range of periodic three movements, and the corresponding interval range has not changed significantly.

\section{Data Availability}

The data used to support the findings of this study are available from the corresponding author upon request.

\section{Conflicts of Interest}

The authors declare that they have no conflicts of interest. 


\section{Acknowledgments}

This work was financially supported by the National Science Foundation (11272190) and the Key Research and Development project of Shandong Province (Public Welfare Projects): Research on Key Technologies of Vehicle Flywheel Battery Vibration Suppression in Complex Environment (2019GGX103024).

\section{References}

[1] Y. G. Luo, S. H. Zhang, and B. B. Wen, "Study on nonlinear characteristics of crack-collision friction coupling in rotorhusband transverse system," Journal of Vibration and Shock, vol. 24, no. 3, pp. 43-48, 2005.

[2] A. Muszynska, "Whirl and whip-rotor/bearing stability problems," Journal of Sound and Vibration, vol. 110, no. 3, pp. 443-462, 1986.

[3] G. Capone, "Orbital motions of rigid symmetric rotor supported on journal bearings," La Meccanica Italiana, vol. 199, no. 1, pp. 37-46, 1986.

[4] G. Capone, "Analytical description of fluid-dynamic force field in cylindrical journal bearing," L'Energia Elettrica, vol. 3, pp. 105-110, 1991.

[5] Z. Y. Jiang, Q. G. Sun, T. J. Li et al., "Bifurcation characteristics analysis of centrifugal pump impeller with loose bearing fault," Journal of Mechanical Strength, vol. 31, no. 5, pp. 707-711, 2009.

[6] J. Zhang and B. X. Wen, "Frequency characteristics analysis of loose rotor system with two end bearings," China Mechanical Engineering, vol. 19, no. 1, pp. 68-71, 2008.

[7] H. Ma, X. Zhao, Y. Teng, and B. Wen, "Analysis of dynamic characteristics for a rotor system with pedestal looseness," Shock and Vibration, vol. 18, no. 1-2, pp. 13-27, 2011.

[8] Y. G. Luo, S. H. Zhang, and B. B. Wen, "Nonlinear dynamic response and chaos of rotor-bearings with double-span rubimpact," Mechanical Science and Technology, vol. 27, no. 11, pp. 1272-1275, 2008.

[9] W. He and H. Q. Yuan, "Analysis of rub-impact fault of doubledisk cantilever rotor-bearing-case-coupled system," Journal of Mechanical Strength, vol. 34, no. 2, pp. 170-175, 2012.

[10] Z. Ren, S. Zhou, C. Li, and B. Wen, "Dynamic characteristics of multi-degrees of freedom system rotor-bearing system with coupling faults of rub-impact and crack," Chinese Journal of Mechanical Engineering, vol. 27, no. 4, pp. 785-792, 2014.

[11] H. Ma, C. Shi, Q. Han, and B. Wen, "Fixed-point rubbing fault characteristic analysis of a rotor system based on contact theory," Mechanical Systems and Signal Processing, vol. 38, no. 1, pp. 137-153, 2013.

[12] A. J. Hu and L. L. Hou, "Dynamic simulation and experimental study of an asymmetric double-disk rotor-bearing system with rub-impact and oil-film instability," Nonlinear Dynamics, vol. 84, no. 2, pp. 641-659, 2016.

[13] Y.-G. Luo, Z.-H. Ren, H. Ma, T. Yu, and B.-C. Wen, "Stability of periodic motion on the rotor-bearing system with coupling faults of crack and rub-impact," Journal of Mechanical Science and Technology, vol. 21, no. 6, pp. 860-864, 2007.

[14] D. Li, H. Q. Yuan, and L. M. Wu, "The nonlinear characteristics of a rotor system with elastic support and two-span rub-impact faults," Journal of Vibration, Measurement \& Diagnosis, vol. 29, no. 4, pp. 415-510, 2009.

[15] G. Z. Liu, Y. Yu, F. Yu et al., "Nonlinear study of rub impact rotor under excitation frequency," Chinese Journal of Construction Machinery, vol. 12, no. 5, pp. 444-449, 2014.
[16] G. Z. Liu, Y. Yu, F. Yu et al., "Rubbing fault study on unsteady oil-film forces for rotor-stator bearing system," Chinese Journal of Construction Machinery, vol. 11, no. 5, pp. 433-437, 2013.

[17] G. Z. Liu, Y. Yu, X. J. Ma et al., "Nonlinear analysis on rub impact rotor with mass eccentricity," Chinese Journal of Construction Machinery, vol. 14, no. 1, pp. 21-26, 2016.

[18] X. Du, Research on Dynamic Modeling and Fault Diagnosis Method for Nonlinear Faulted Rotor System, Hunan University, Changsha, Hunan, China, 2008. 


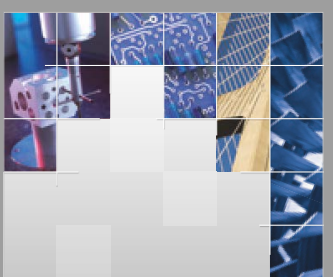

\section{Enfincering}


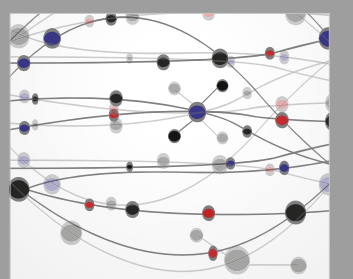

\section{Rotating \\ Machinery}

The Scientific World Journal

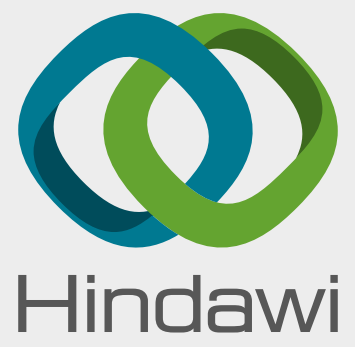

Submit your manuscripts at

www.hindawi.com
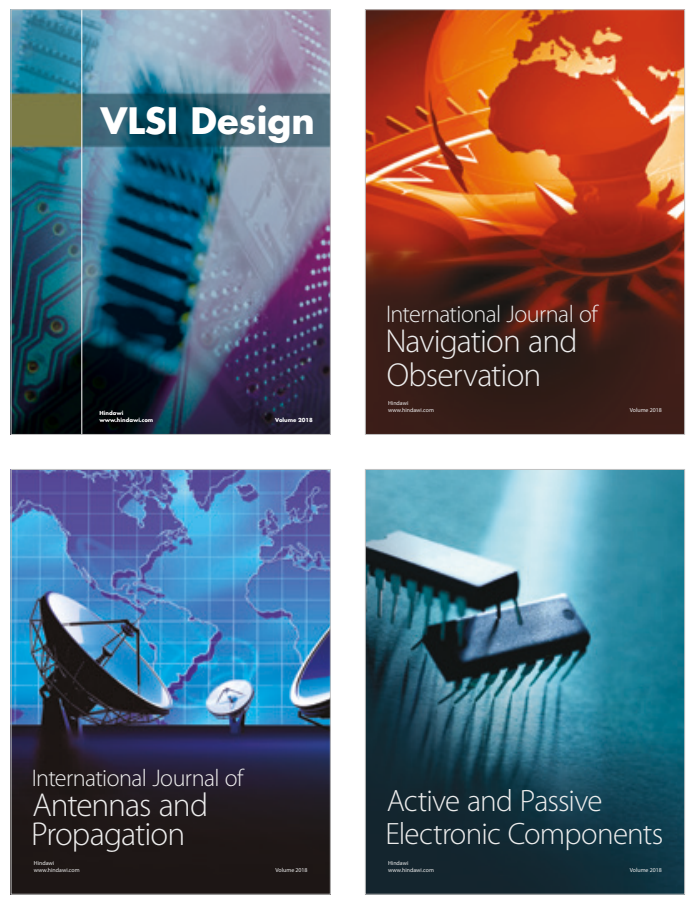
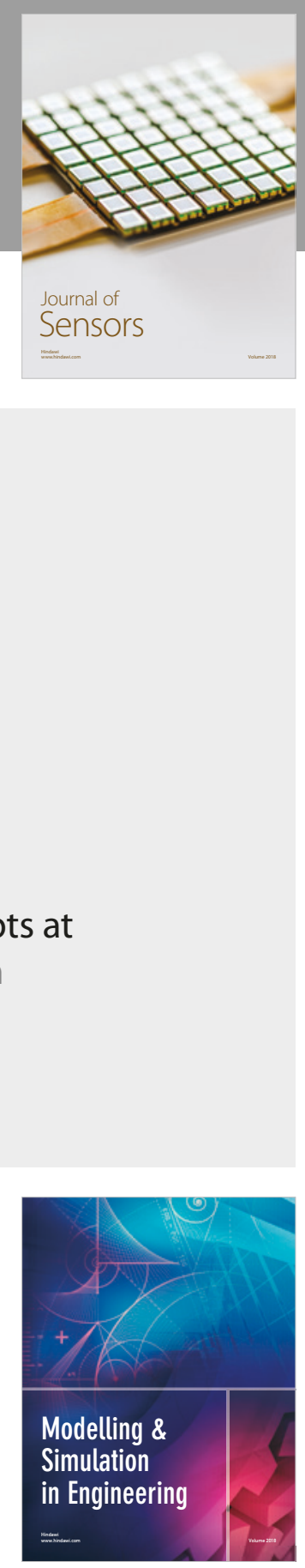

\section{Advances \\ Multimedia}
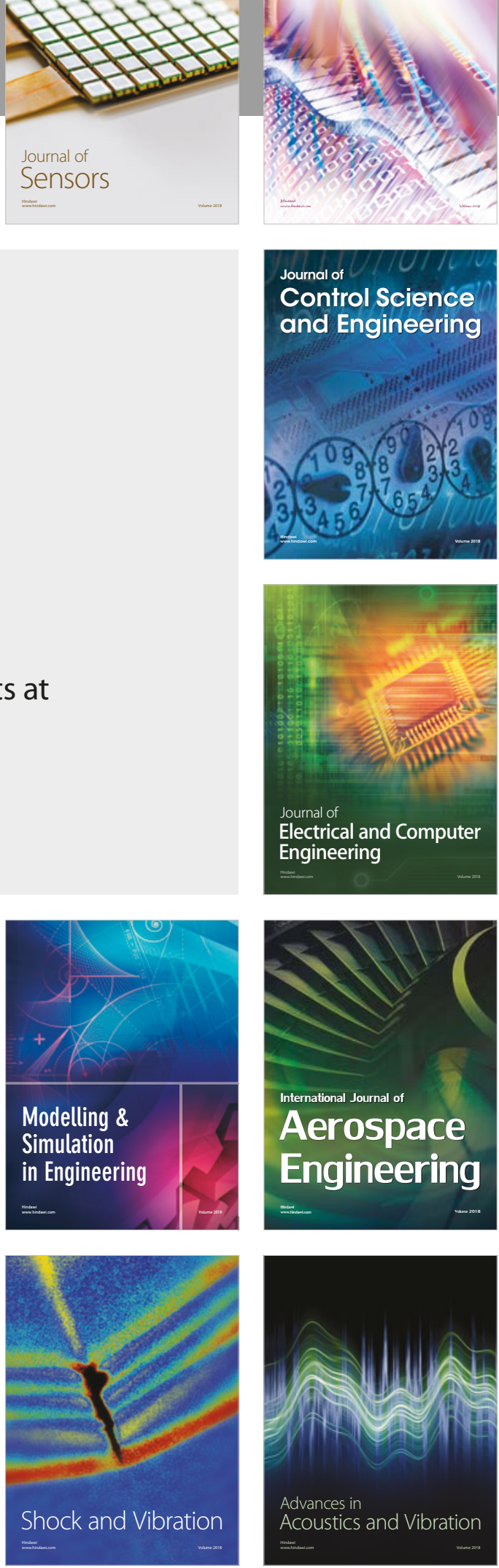